\title{
ESTRELLAS MÁGICAS Y ECUACIONES LINEALES
}

\author{
Stars magical and linear equations
}

MARCO VINICIO VÁSQUEZ BERNAL*

Recibido: 11 de Noviembre de 2015. Aceptado: 30 de Noviembre de 2015

DOI: http://dx.doi.org/10.21017/rimci.2016.v3.n5.a8

\section{Resumen}

Entre las relaciones matemáticas curiosas, se tiene las estrellas mágicas, que consiste en estructuras geométricas, con la forma de estrellas, con números ubicados en los puntos de corte de los lados, y cuya suma por segmento es constante.

Palabras clave: estrellas, estructuras, ecuaciones, lineales.
\end{abstract}

\section{Abstract}

Among the curious mathematical relations have magical stars, which consists of geometric structures in the shape of stars, with numbers located on the cutoffs of the sides, and whose sum per segment is constant.

Keywords: stars, structures, equations, linear.

\section{INTRODUCCIÓN}

Estrellas MáGicas de cinco puntas [1], para entender esta definición, primero debemos explicar los elementos que se identifican en una estrella, se puede observar en la Fig.1.

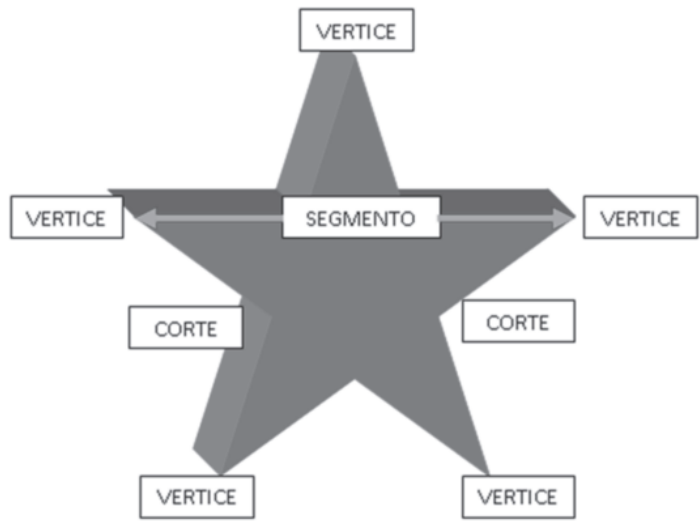

Fig. 1. Estrella de cinco puntas.
En la Fig. 1 tenemos una estrella de cinco puntas y se puede ver claramente lo que llamaremos, vértices, que son los cortes en los extremos de dos lados, coincidiendo con lo que comúnmente se conocen como puntas de las estrellas. Corte define al punto de intersección entre dos lados en ubicaciones intermedias de los extremos. Segmento en cambio es la recta que une dos vértices. Consecuentemente cada segmento contiene cuatro puntos, dos vértices y dos cortes, Además se puede observar que una estrella de cinco puntas, esta constituida por cinco segmentos, y todas ellas se cortan en algún punto, ya sea vértice o corte [2-4].

En la Fig. 2 se observan todos los elementos que son parte de una estrella de cinco puntas, se tienen cinco vértices y cinco cortes, lo que da diez puntos. Es en esos diez puntos donde se deberán ubicar los valores que cumplan las diversas condiciones [5].

* Matemático, mención estadística. Magíster en Gerencia Empresarial (MBA). Magíster en Investigación para el Desarrollo Educativo. Diplomado Superior en Práctica Docente Universitaria y Especialista en Educación Universitaria. Actualmente, catedrático de la UNAE (Universidad Nacional de Educación de Ecuador). Miembro del Consejo editorial de la UNAE. Miembro del Consejo Editorial de la revista Mamakuna. Delegado Embajador de Ecuador en el Parlamento Internacional de Educación. Columnista de El Heraldo del Cañar y de Ecuadoruniversitario.com. Correo electrónico: marco.vasquez@unae.edu.ec 


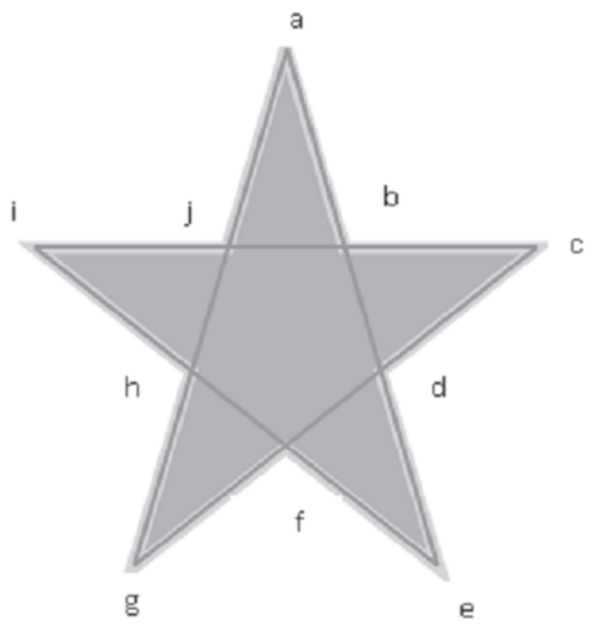

Fig. 2. Estrella con elementos.

En la Fig. 3, se observa las distintas relaciones que existen entre vértices, Cortes y segmentos.

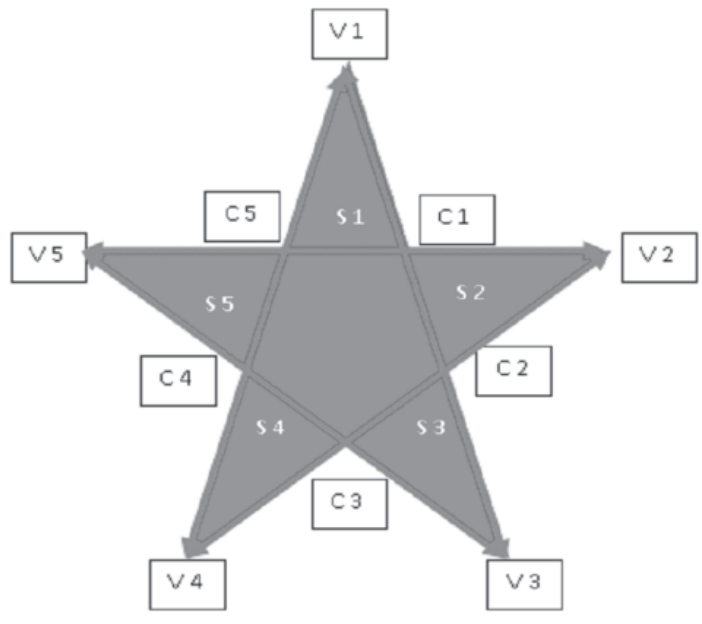

Fig. 3. Estrella con segmentos.

Se observa claramente que:

1) El segmento 1 contiene $\mathrm{V} 5, \mathrm{C} 5, \mathrm{C} 1$ y V2.

2) El segmento 2 contiene $\mathrm{V} 1, \mathrm{C} 1, \mathrm{C} 2 \mathrm{y} \mathrm{V} 3$.

3) El segmento 3 contiene $\mathrm{V} 2, \mathrm{C} 2, \mathrm{C} 3$ y V4.

4) El segmento 4 contiene V3, C3, C4 y V5.

5) El segmento 5 contiene V4, C4, C5 y V1.

Tomando en cuenta las relaciones expuestas de la Fig. 2 se tiene que:

1) El segmento 1 contiene los puntos $i, j, b$ y c.

2) El segmento 2 contiene los puntos a, b, d y e.

3) El segmento 3 contiene los puntos $g$, f, d y c.

4) El segmento 4 contiene los puntos i, h, f y e.

5) El segmento 5 contiene los puntos $g, h, j y a$.

\section{Propiedades DE LAS ESTRELLAS MÁGICAS}

Basándonos en la definición de estrellas mágicas se tiene que las condiciones a las que la misma debe sujetarse genera el siguiente sistema de ecuaciones:

$$
\begin{aligned}
& i+j+b+c=a+b+d+e \\
& i+j+b+c=g+f+d+c \\
& i+j+b+c=i+h+f+e \\
& i+j+b+c=g+h+j+a
\end{aligned}
$$

Sistema de ecuaciones

Que se reduce al siguiente:

$$
\begin{aligned}
& i+j+c=a+d+e \\
& i+j+b=g+f+d \\
& j+b+c=h+f+e \\
& i+b+c=g+h+a
\end{aligned}
$$

Sistema de ecuaciones

Donde tenemos un sistema de cuatro ecuaciones con diez incógnitas, cuya resolución es posible si ingresamos seis condiciones o valores.

Lo que teóricamente posibilita que ingrese seis valores y busque los demás.

\section{A. Caso 1. Soluciones con números consecutivos}

Trataremos de construir una estrella mágica con números del 1 al 10. Lo primero que deberemos calcular es cuánto deben sumar los elementos en cada uno de los segmentos, para ello, sabemos que la suma de los diez primeros números es 55 , y además sabemos que cada número esta presente en dos segmentos por lo que el total de las sumas en los cinco segmentos será 110 , como además, tenemos cinco segmentos, cuyos elementos deben sumar un valor constante, en consecuencia cada segmento debe contener cuatro elementos cuya suma será siempre 22 .

A continuación indicaremos todas las posibles muestras de cuatro elementos de enteros entre $1 \mathrm{y}$ 10 , cuya suma total es 22 .

$(1,2,9,10),(1,3,8,10),(1,4,7,10),(1,4,8,9)$, $(1,5,6,10),(1,5,7,9),(1,6,7,8),(2,3,7,10),(2,3,8,9)$,

Rev. Ingeniería, Matemáticas y Ciencias de la Información Vol. 3 / Núm. 5 / enero-junio de 2016; pág. 79-97 
$(2,4,6,10),(2,4,7,9),(2,5,6,9),(2,5,7,8),(3,4,5,10)$, $(3,4,6,9),(3,4,7,8),(3,5,6,8),(4,5,6,7)$.

Para lograr el objetivo planteado, deberemos seleccionar cinco de estos, de tal forma que cada número debe estar presente en dos de ellos, intentamos pero no es posible. Como ejemplo tomemos el cuarteto $(1,2,9,10)$, el siguiente debe ser uno en el cual coincida únicamente un elemento, cumple el $(1,6.7 .8)$, coincidiendo el 1 . El tercer cuarteto seleccionado debe no contener el $1 \mathrm{y}$ además incluir un elemento de cada uno de los dos, que sean distintos del 1, y los demás deben diferir de los otros dos de los cuartetos seleccionado, escogemos $(3,4,6,9)$, que coincide con el primero en el 9 , con el segundo tienen en común el 6 , y no tiene ni 1 , ni 2 ni de 10 que están en el primero, ni 7, ni 8 que están en el segundo. El siguiente cuarteto, el cuarto debe contener uno de los elementos del primero, segundo y tercero, que aún no sean coincidentes, para a continuación ubicar el elemento que debe ser nuevo y cuya suma debe ser 22:

1) Si tomamos $2,7,3$, el cuarto elemento debería ser 10 que no es nuevo.

2) Si tomamos $2,7,4$, el cuarto elemento es 9 que no es nuevo.

3) Si tomamos 2,8,3, el cuarto elemento es 9 que no es nuevo.

4) Si tomamos 2,8,4, el cuarto elemento es 8 que no es nuevo.

5) Si tomamos 10,7,3, el cuarto elemento es 2 que no es nuevo.

6) Si tomamos 10,7,4 el cuarto elemento es 1 que no es nuevo.

7) Si tomamos 10,8,3, el cuarto elemento es 1 que no es nuevo, $y$,

8) Si tomamos $10,8,4$, la suma ya es 22 , no hay posible cuarto elemento.

Por tanto no es posible construir la estrella mágica con ese tercer cuarteto, no existen otros cuartetos que puedan cumplir como terceros y no existe otro que cumpla para ser segundo. Se podría pensar que no se construya en función del 1 sino del 2 y tendríamos:

1) $1^{\circ}$ cuarteto $(1,2,9,10)$

2) $2^{\mathrm{a}}$ cuarteto $(2,5,7,8)$

3) $3^{\text {a }}$ cuarteto $(3,4,5,10)$
Para el cuarto, los que se repiten ya son $2,5 \mathrm{y}$ 10, entonces podemos tomar:

1) $1,7,3$, el cuarto sería 11 , que no es elemento.

2) $1,7,4$, el cuarto sería 10 , que no es nuevo.

3) $1,8,3$, el cuarto sería 10, que no es entero.

4) $1,8,4$, el cuarto sería 9 , que no es nuevo.

5) $2,7,3$, el cuarto sería 10, que no es nuevo.

6) 2,7,4, el cuarto sería 9 , que no es nuevo.

7) $2,8,3$, el cuarto sería 9 , que no es entero.

8) $2,8,4$, el cuarto sería 8 , que no es nuevo.

Por tanto tampoco es posible. Si intentamos construir en función del 6 tendríamos:

1) $1^{\circ}$ cuarteto $(1,6,7,8)$

2) $2^{\text {a }}$ cuarteto $(3,4,6,9)$

3) $3^{\text {a }}$ cuarteto $(1,2,9,10)$

Para el cuarto, los que se repiten ya son 1,6 y 9 , entonces podemos tomar:

1) $7,3,2$ el cuarto sería 10 , que no es nuevo.

2) $7,3,10$, el cuarto sería 2 , que no es nuevo.

3) $7,4,2$, el cuarto sería 9 , que no es entero.

4) $7,4,10$ el cuarto sería 1 , que no es nuevo.

5) $8,3,2$ el cuarto sería 9 , que no es nuevo.

6) $8,3,10$ el cuarto sería 1 , que no es nuevo.

7) $8,4,2$, el cuarto sería 8 , que no es entero.

8) $8,4,10$ el cuarto sería 0 , que no es elemento.

Con lo que observa que tampoco así es posible, así podemos intentar varias veces y no podremos construir la estrella mágica deseada, lo que lleva a afirmar que No es posible construir una estrella magica con los números naturales del 1 al 10.

En consecuencia de lo anterior se puede afirmar que: no es posible construir una estrella magica con los numeros consecutivos ni con los multiplos sucesivos de cualquier número.

\section{B. Caso 2. Construcción de estrellas mágicas en función de una variable}

En este caso lo que intentaremos será obtener los distintos elementos a partir o en función de una variable. Esto es posible si tomamos en cuenta las características de estrella mágica, esto es que todos las sumatorias de los elementos en cada segmente brinda un resultado único, y partiendo de un elemento, construimos los demás, debiendo 
ubicarse el ultimo elemento que debe satisfacer la condición de igualdad para los dos segmentos al que pertenece, cuyos resultados deben darme una ecuación soluble, no una contradicción, de ser una contradicción, esa estrella mágica no es posible, de obtenerse una identidad significaría que existe una estrella por cada valor asignado a la variable y si se tiene un ecuación soluble, la respuesta de la ecuación es el valor que hace que sea posible construir la ESTRELLA MÁGICA.

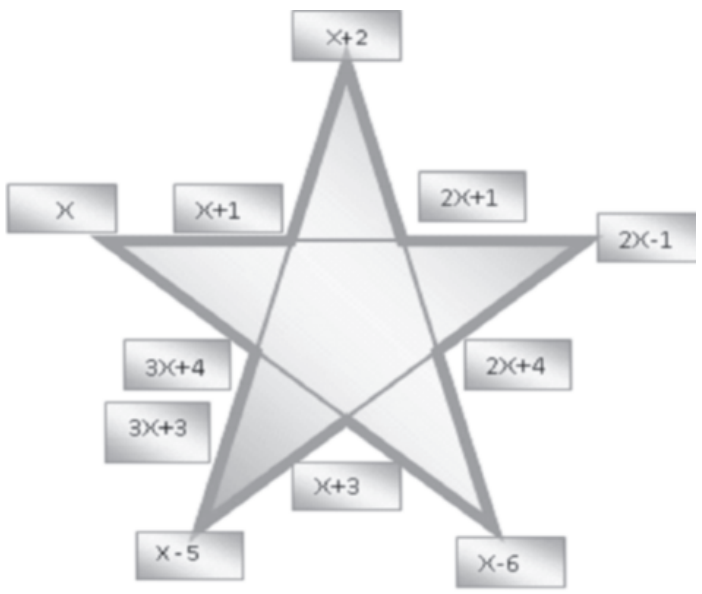

Fig. 4. Estrella en función de una variable.

Ejemplo 1. En este caso iniciamos ubicando la variable $X$ en un vértice, luego en el corte ubica$\operatorname{mos} X+1$, en el siguiente corte ubicamos $2 X+1$ y en el otro vértice de ese segmento ubicamos $2 X-1$, obteniendo el total del segmento de $6 \mathrm{X}+1$, colocamos otros vértices y cortes, cuidando que sean distintos entre si y que guarden la relación de que su sumatoria sea constante, así hasta llegar al último elemento, en este caso un corte, que según el un segmento debe ser $3 X+4$ y según el otro al que pertenece $3 X+3$. Si igualamos estos dos valores tenemos la igualdad: $3 X+3=3 X+4$, lo que es una contradicción, en consecuencia no es posible construir una estrella mágica con esos valores.

En otros casos tendremos que el valor obtenido, repite alguno de los asignados, tampoco se podrá crear esa estrella.

NOTA: El orden de asignar los valores es arbitraria y personal, lo que se siguiere es primero llenar un segmento para obtener la constante de la suma, luego ubicar los siguientes valores hasta que una relación de igualdad en un elemento genere una ecuación soluble, cuyo resultado permite construir la ESTRELLA MÁGICA.

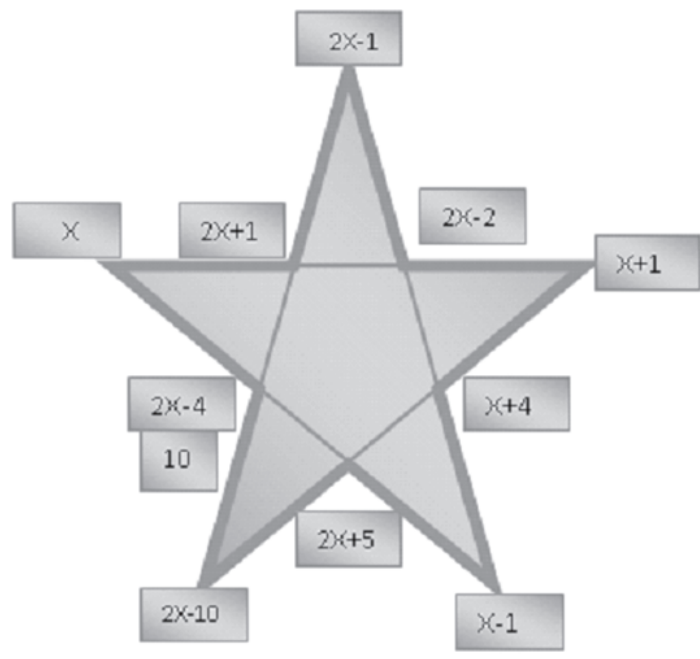

Fig. 5. Estrella con total del segmento de $6 X+1$

Ejemplo 2. Se tiene la siguiente estructura, donde se observa que los elementos de todos los segmentos suman 6X, de ello se desprende la siguiente igualdad $2 X-4=10$, que brinda como solución cuando $X=7$, que remplazando permite construir la siguiente estrella mágica de cinco puntas: Donde todos los segmentos suman 42 .

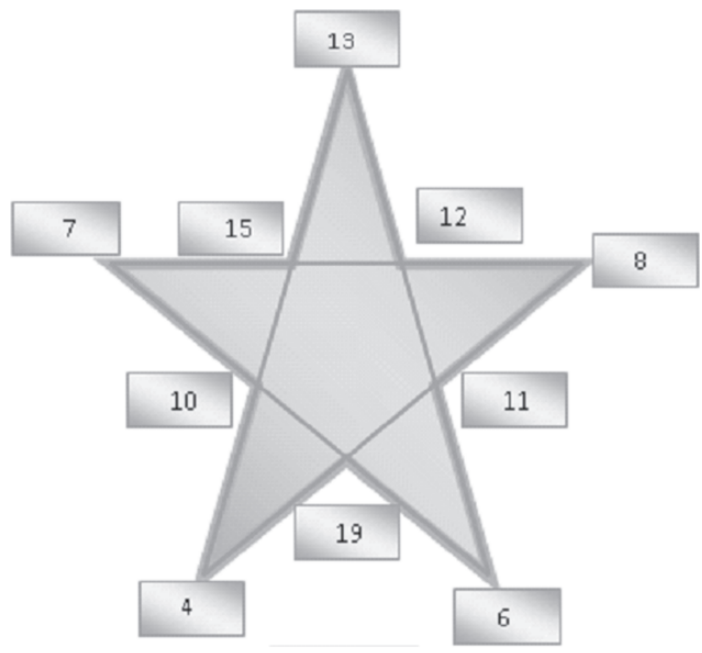

Fig. 6. Estrella con todos los segmentos que suman 42 .

Ejemplo 3. Aquí construimos una estructura cuya constante de suma es $2 X+5$, la igualdad se genera en un vértice.

De donde se tiene en vértice 4 , de los resultados de los segmentos a los que estos representan $X+4=-X+3$, cuya solución es $X=-0.5$, con lo cual tenemos la estrella mágica de la Fig. 7. La constante de suma en los segmentos es 4 .

Rev. Ingeniería, Matemáticas y Ciencias de la Información Vol. 3 / Núm. 5 / enero-junio de 2016; pág. 79-97 


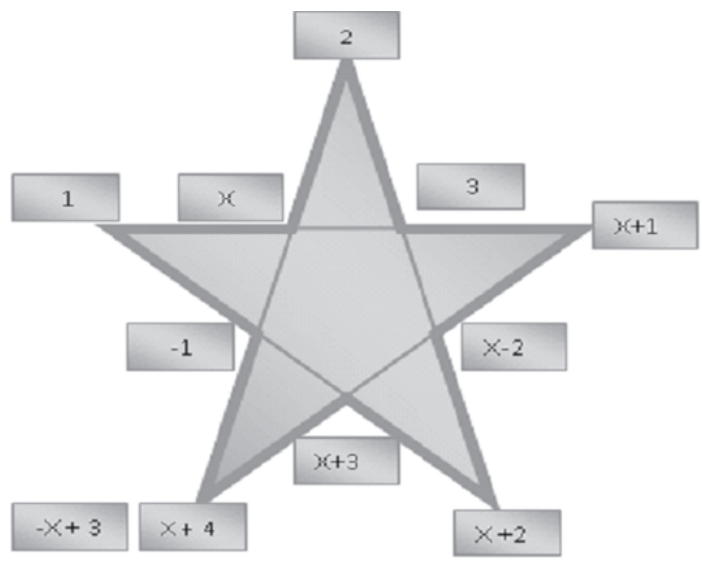

Fig. 7. Estrella con constante de suma 4.

NOTA: Por simple análisis se puede decir que si a todos los elementos de una estrella mágica le sumamos un valor constante o le multiplicamos por un valor constante, su respectivos resultados generan también una estrella mágica.

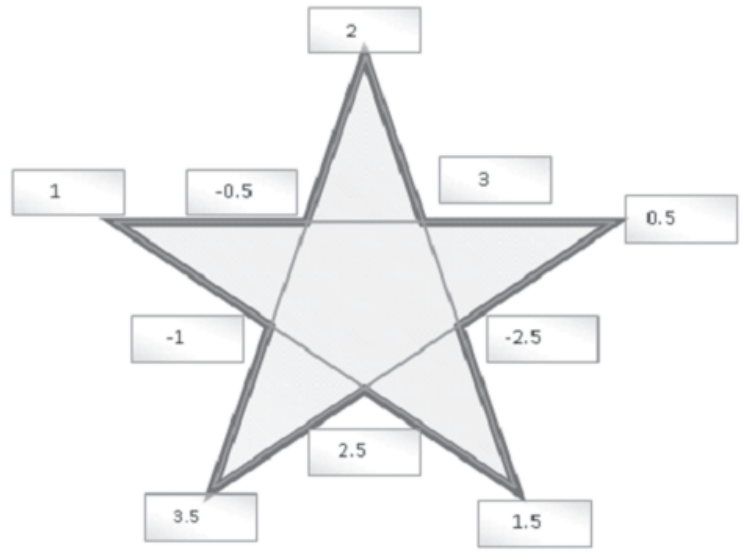

Fig. 8. Resulado Estrella con constante de suma 4.

Así si a la estrella de la Fig. 8, multiplicamos cada elemento por 2 y sumamos 6 tendremos:

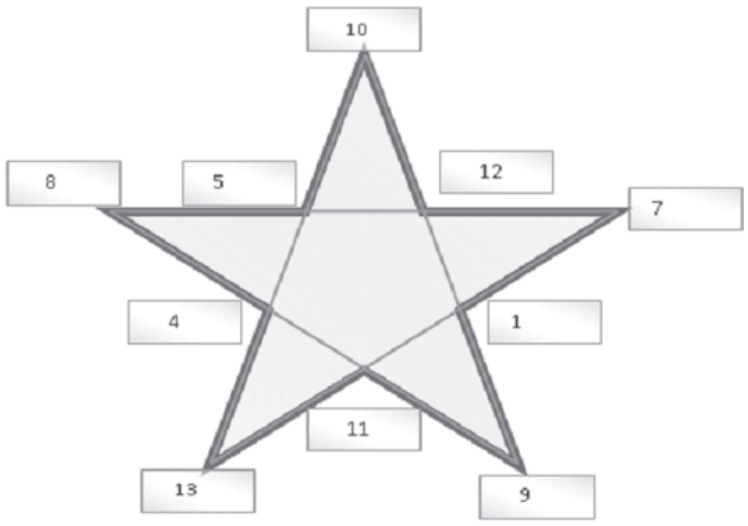

Fig. 9. Estrella con cada elemento multiplicado.
Donde la constante de suma es 32. Ya que inicialmente sumaba 4 al multiplicar por 2 tendríamos 8 y luego sumamos 6 a cada elemente, es decir sumamos 24 a cada segmento, lo que me da un aumento total de 32 por segmento.

También si sumamos los elementos respectivos de dos estrellas mágicas, el resultado es otra estrella mágica, donde la constante de suma en sus segmentos es el resultado de sumar las dos constantes de sumas de las dos primeras estrellas mágicas.

Sumemos las estrellas mágicas de la Fig. 8 y la Fig. 9, tendremos el resultado presentado en la Fig. 10.

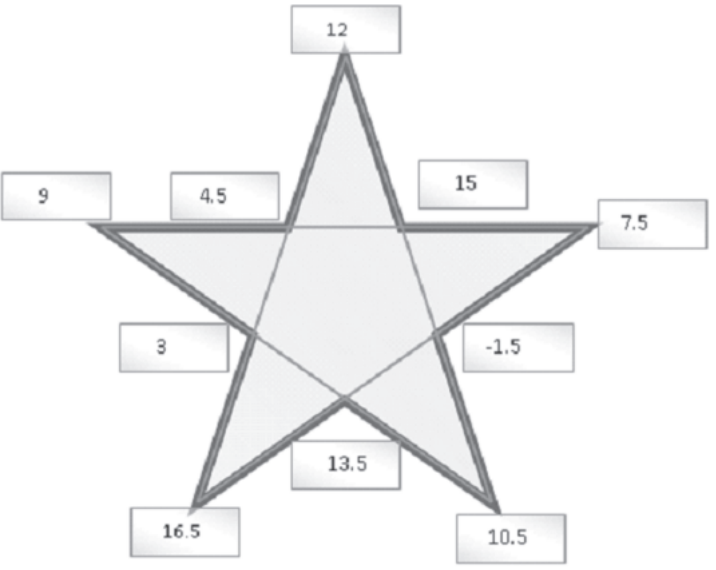

Fig. 10. Estrella suma de Fig. 8 y Fig. 9.

Cuya constante de suma, que es 36 es el resultado de sumar la constante de suma de la Fig. 8, que es 4, más la constante de la figura 9 que es 32 .

Si deseamos que los elementos sean todos positivos y enteros, deberíamos multiplicarlos por 2 y sumar 4, y tendríamos una estrella mágica con constante de suma 88.

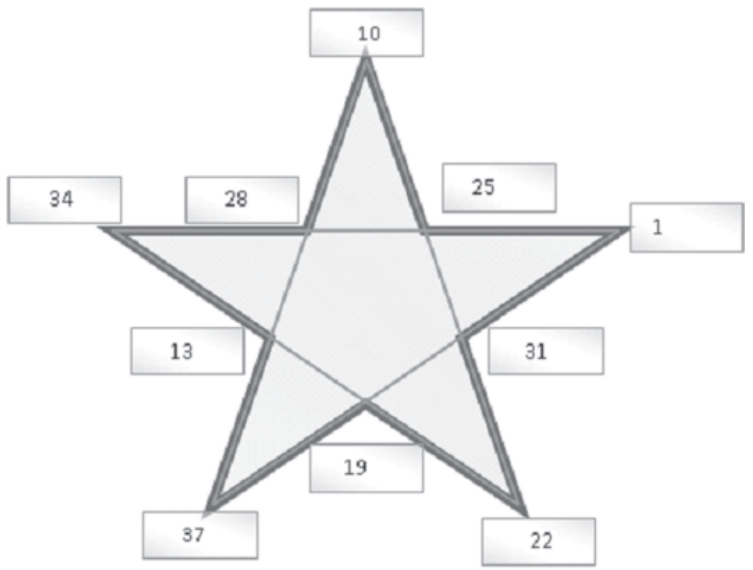

Fig. 11. Estrella con elementos enteros. 


\section{DeRIVADAS DE UNA ESTRELla DE CINCO PUNTAS}

$\mathrm{Al}$ tener ya construida una estrella mágica, se pueden generar otras con una reubicación de los elementos, obviamente que se mantendrá los elementos de cada segmento, pudiendo cambiarse entre vértices y cortes. Un proceso sencillo es fijar un vértice, dos segmentos parten de ese punto y los tres se cortan con estos en distintas secciones, tomamos de estos tres segmentos los pares que cortan con los dos fijos y permutamos entre estos, eso me da 6 estrellas mágicas en cada caso, además a cada estrella mágica se le puede construir su simétrica, que obtiene únicamente girando ciento ochenta grados la estrella. Con lo indicado, fijando un vértice se puede generar 12 estrellas mágicas, como se observa en la Fig. 12.

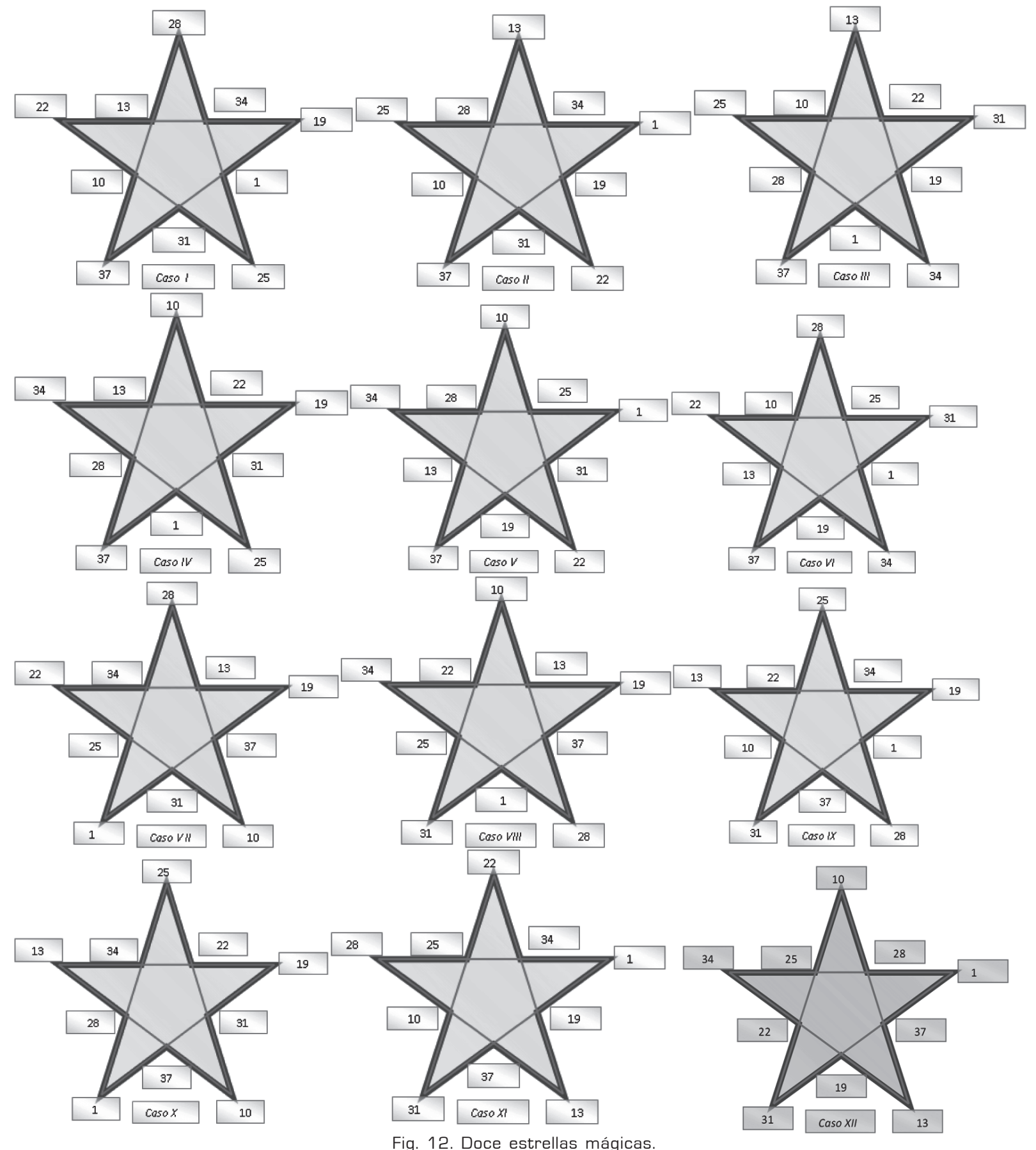

Rev. Ingeniería, Matemáticas y Ciencias de la Información Vol. 3 / Núm. 5 / enero-junio de 2016; pág. 79-97 
En estos doce casos se tienen todas las posibles variaciones, debiendo indicar que para cada uno de ellos se puede generar otro simétrico, con lo cual tendríamos que con los mismos elementos es posible generar 24 estrellas mágicas.

Este resultado coincide además con el número de permutaciones de las cuatro combinaciones que se relacionan con otra combinación fija, ya que $4 !=24$, obviamente en este cálculo estarán las simétricas. Además teniendo en cuenta las posibles rotaciones en total tendremos 120 estrellas mágicas o las permutaciones de las cinco combinaciones $(5 !=120)$.

\section{A. Caso 3. Construcción de estrellas mágicas de cinco puntas con dos variables}

Este proceso, brinda una gama mayor de respuestas, si bien se sujete a los mismos lineamientos, sus resultados ya no son únicos, son determinados mas bien por relaciones lineales entre las dos variables, cuyos resultados son infinitos pares, lo que aquí se deberá cuidar más bien es que al asignar los valores, estos sean únicos.

Ejemplo 4. Iniciamos construyendo esta estrella mágica ubicando en un vértice la variable $X$ y en un corte aledaño, la variable $Y$, luego ubicamos valores, en función de las variables en los distintos elementos, de tal forma que la sumatoria constante sea $3 X+3 Y$, para el ultimo elemento, obtenemos dos posibles resultados, $2 Y-1$, según el un segmento y $\mathrm{X}+\mathrm{Y}-1$, según el otro segmento. Cumpliendo la condición de igualdad se tendrá que:

$2 Y-1=X+Y-1$, que se simplifica a: $X=Y$, condición que muestra que este caso irrespeta la unicidad de los elementos, por tanto este caso no genera estrella mágica alguna.

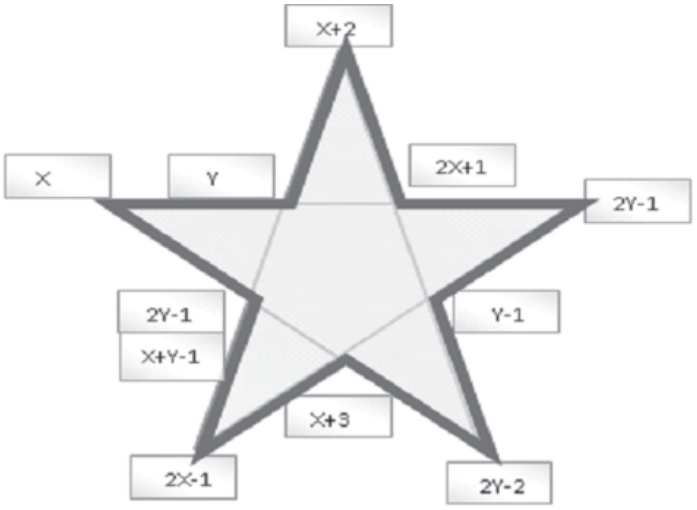

Fig. 13. Estrella mágica con dos variables.
Ejemplo 5. Siguiendo el mismo orden anterior, construimos la siguiente estrella mágica, a nivel de variables, de aquí se obtiene la relación; $2 Y-5=X$ $+Y+3$, Que se simplifica a: $Y-X=8$. Aquí tenemos entonces una relación lineal entre $\mathrm{X}$ e $\mathrm{Y}$.

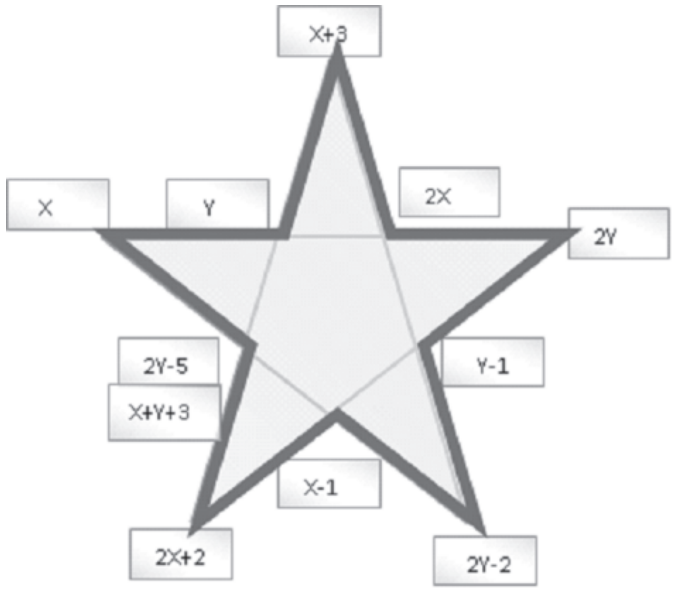

Fig. 14. Estrella - Relación lineal.

Son infinitas las duplas que cumplen esta relación, ponemos indicar algunas de ellas, tomando únicamente valores enteros, aun que se puede tomar en cualquier conjunto de números donde se cumpla la igualdad: $(-3,5),(-2,6),(-1,7),(0,8),(1,9)$, $(2.10),(3,11)$, etc.

Lo que a continuación debemos ver es si remplazando los valores de estas duplas en cada elemento propuesto, se da la unicidad.

Veamos por ejemplo cuando $\mathrm{X}=-3$ e $\mathrm{Y}=5$.

Se observa en la Fig. 15 que se repiten dos -4 y dos 5, por tanto debemos intentar con otra dupla.

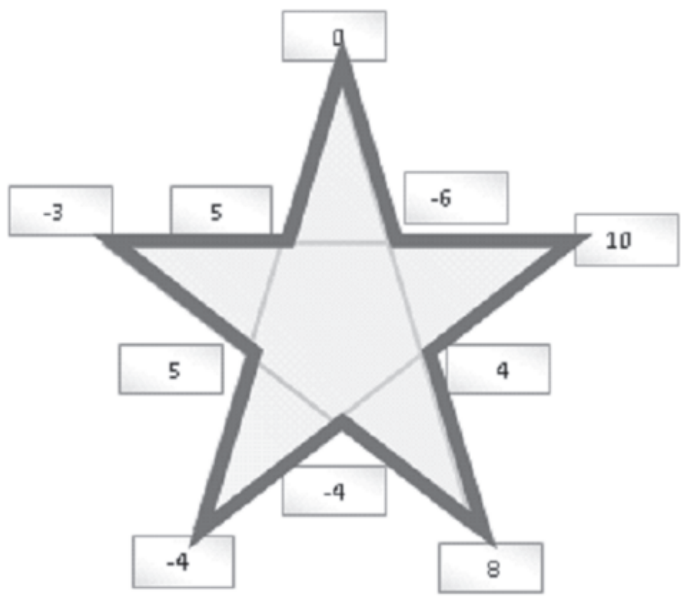

Fig. 15. Estrella - Reemplazando valores. 
Tomamos otros valores $\mathrm{X}=2, \mathrm{Y}=10$, con los cuales tendríamos la siguiente estrella:

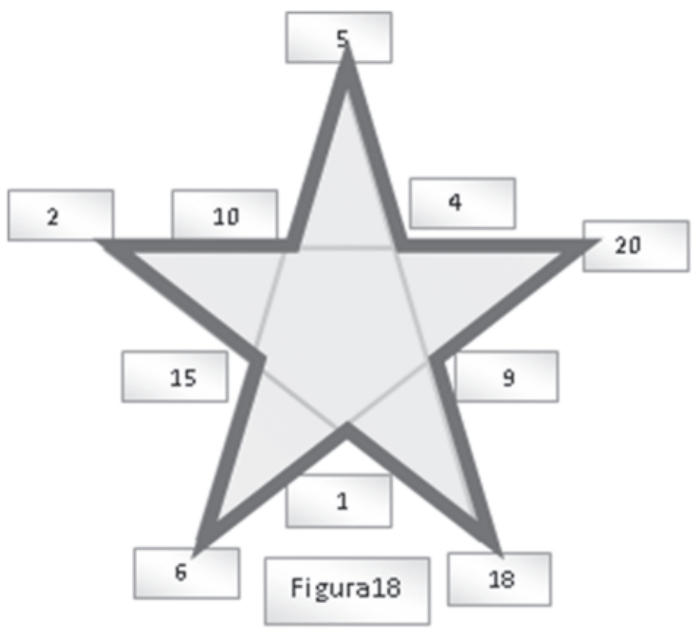

Fig. 16. Estrella $-X=2, Y=10$.

De la Fig. 16 se observa que la sumatoria constante es 36 y además cada elemento esta presente una sola vez, por tanto esta si es una Estrella Mágica, y podremos derivar otras respectivas.

\section{B. Caso 4. Construcción de estrellas mágicas de cinco puntas con tres variables}

Proponemos esto, como una generalización de lo expuesto, en este caso intentaremos construir estrellas mágicas basándonos en tres variables, X, Y e Z, para este caso. Asignando valores tendríamos:

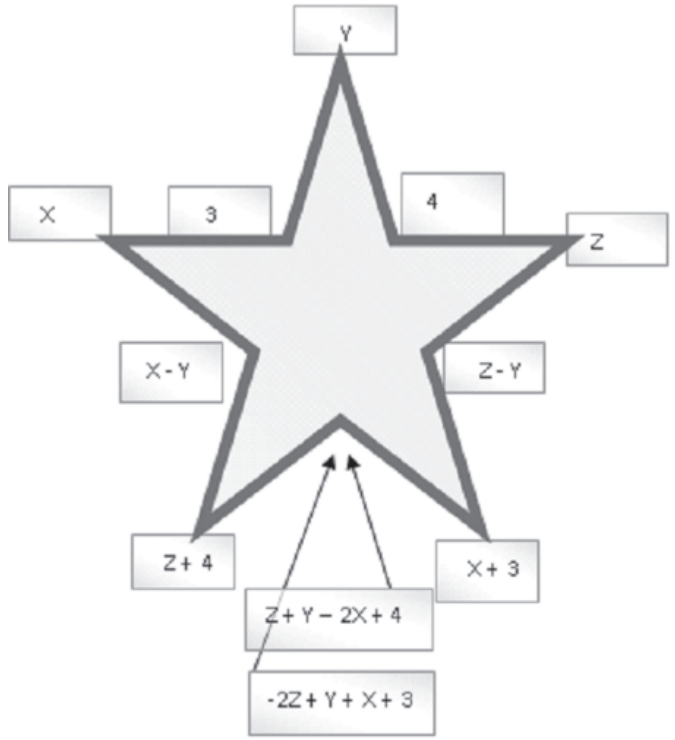

Fig. 17. Estrella mágica con tres variables.
Ejemplo 6. Iniciamos construyendo esta estrella mágica ubicando en tres vértices las variables X, Y e Z, en los cortes aledaño, ubicamos 3 y 4 , lo que posibilita que sobre un eje encontremos $X+Z+7$, como la constante de suma, en los otros vértices ubicamos $X+3$ y $Z+4$, en los dos vértices ubicamos Z-Y e X - Y, que cumple la constante de suma quedando pendiente un el ultimo corte, que como forma parte de dos segmentos, debe ser igual a $Z+Y-2 X+4$ para formar parte del primer segmento y $-2 Z+Y+X+3$, por ser parte del otro segmento. Con lo que tendríamos la siguiente igualdad:

$Z+Y-2 X+4=-2 Z+Y+X+3$, que simplificando se tiene $Z=(-1+3 X) / 3$. O lo que es lo mismo: $Z$ $=\mathrm{X}-1 / 3$.

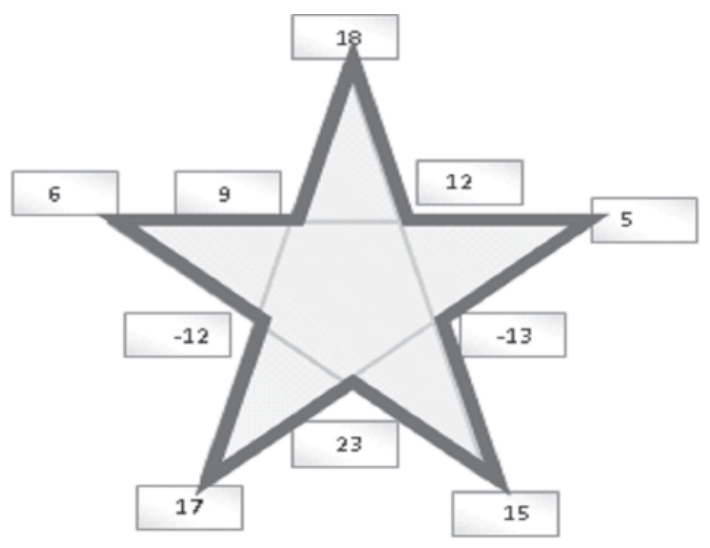

Fig. 18. Estrella mágica valores enteros.

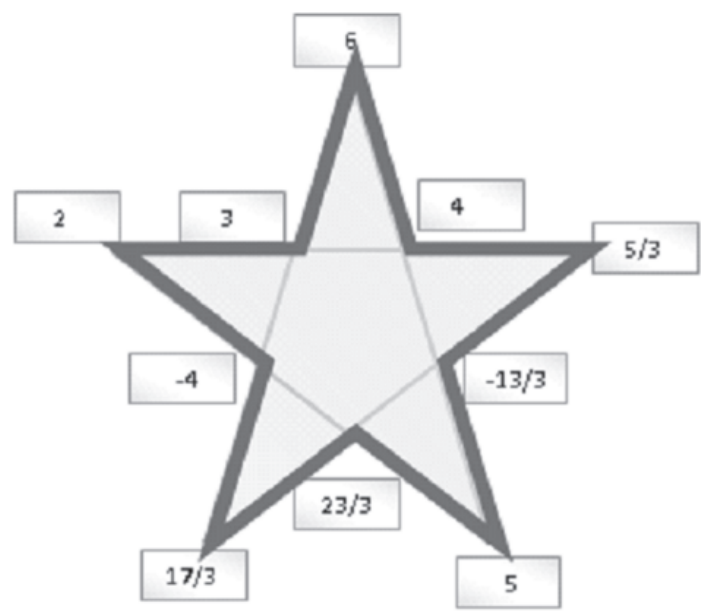

Fig. 19. Estrella mágica 32/3. 


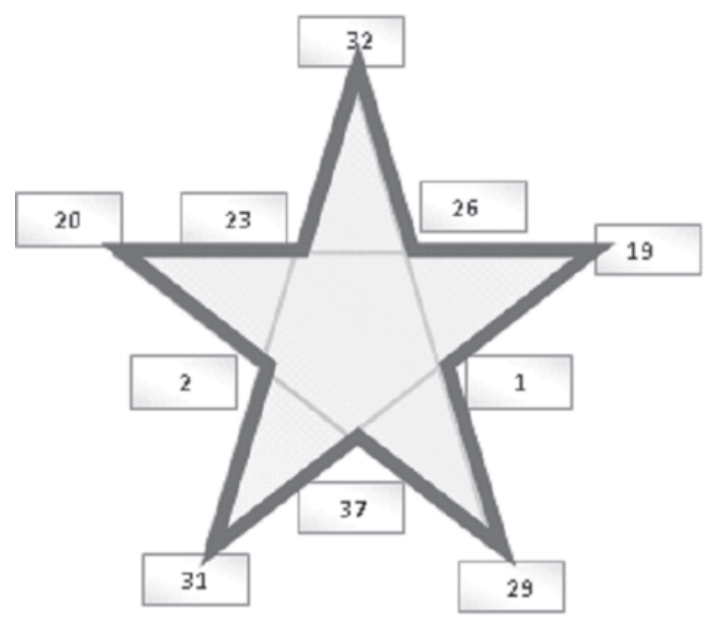

Fig. 20. Estrella sumar 14 de la Fig. 18.

Así podremos asignar valores a $X, Y, Z$ que se sujeten a esa relación, se observa que la relación es entre $\mathrm{X}$ y $\mathrm{Z}$, por tanto $\mathrm{Y}$ se puede asignar arbitrariamente, así vamos a asignar los valores siguientes: $X=2, Y=6, y Z=5 / 3$.

Con lo cual podemos construir la Fig.17, que satisface las condiciones de estrella mágica, aun que estén presentes valores negativos y fraccionarios, si multiplicamos todos los valores por tres, tendremos la Fig. 18, con valores enteros, y si además sumamos 14 a todos los valores, tendremos la Fig.20, esta claro que cambiara la constante sumatoria, en la Fig. 19 es 32/3, en la Fig.18 es 32 y en la Fig. 20 es 88.

\section{Caso 5. Construcción de estrellas mágicas de cinco puntas con cuatro variables}

Ejemplo 7. Para este caso fijaremos cuatro variables $X, Y, Z$ y $W$, en cuatro vértices, como se observa en la Fig. 21, en cortes entre $X$ e $Y$, ubicamos la constante 2 , entre $Y$ y $Z$, ubicamos la constante 4 , además entre los vértices $W$ y el no asignado, ubicamos la constante 5 , teniendo como constante de suma el valor de $X+Z+6$, obligando ubicar $X+Z-W-Y+2$, en el corte entre $Z$ y $W$, además de deberá ubicar $-Z+W+Y-1$, en el vértice que no tiene valor, concluyendo que el ultimo vértice, en función de la constante de la suma tendremos los dos valores, por un lado: $X-W-$ $2 Y+2 Z+5$, y por otro $-W+Z+1$, en función de las igualdades que deben darse en los segmentos:

Por tanto tenemos la igualdad $\mathrm{X}-\mathrm{W}-2 \mathrm{Y}+2 \mathrm{Z}+5=$ $-W+Z+1$
Que se resume a: $Z+X-2 Y+4=0$.

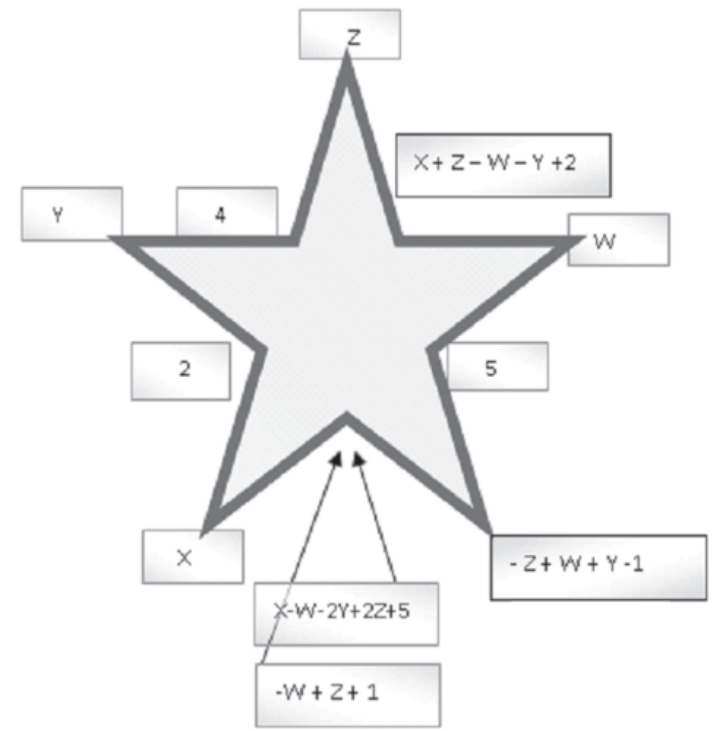

Fig. 21. Estrella mágica de 4 variables.

Esta relación se da entre las variables X, Y e Z, posibilitando que $\mathrm{W}$ sea asignado arbitrariamente. Con lo indicado asignamos los siguientes valores: $Z=0, X=-6, Y=-1$ y $W=5,5$.

Se observa que la sumatoria por segmento será 0 , y se construye la Fig. 22, que tiene valores reales y negativos, si deseamos una estrella mágica con valores positivos y enteros, multiplicaremos cada elemento por 2 y sumando 18, obteniéndose la Fig. 23, cuya constante de suma por segmento será 72 .

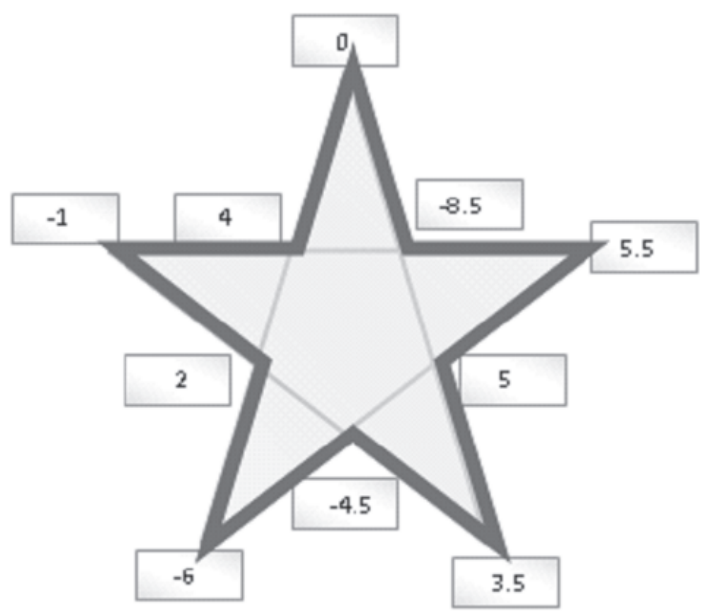

Fig. 22. Estrella mágica valores reales y negativos. 


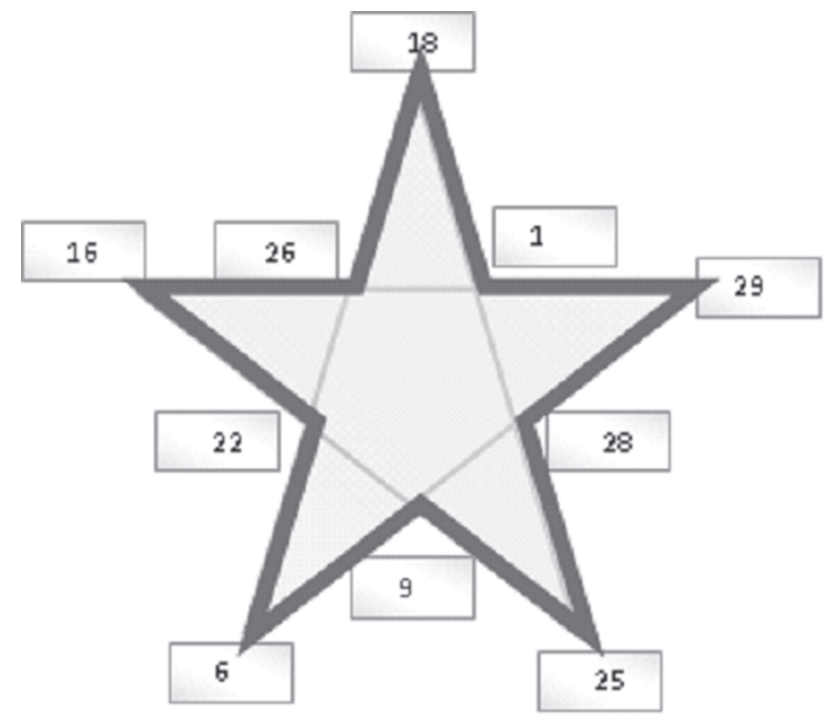

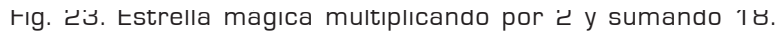

Está claro que tanto las estrellas expuestas no son únicas en ninguna caso, aquí lo que expone es simplemente una forma de construirlas, sabiendo también que para cada una existen las 12 derivadas.

\section{Caso 6. Construcción de estrellas mágicas con cinco variables}

El procedimiento es similar a los anteriores, y las limitaciones también, propondremos un caso donde ubicaremos las variables en las puntas de las estrellas, las variables a utilizarse serán: $X, Y$, $\mathrm{Z}, \mathrm{W}, \mathrm{U}$, resultando el siguiente ejemplo:

Ejemplo 8. En la Fig. 24, se establece los valores para cada uno de los elementos en función de las variables; $\mathrm{X}, \mathrm{Y}, \mathrm{Z}, \mathrm{W}, \mathrm{y} \mathrm{U}$ :

Se tiene que la constante por cada segmento es $\mathrm{X}+\mathrm{Y}+\mathrm{Z}+\mathrm{U}+2$, Donde en uno de los puntos de corte se tiene la igualdad:

$-\mathrm{X}-\mathrm{W}+\mathrm{U}+\mathrm{Z}+\mathrm{Y}+1=\mathrm{W}+2 \mathrm{Y}-2 \mathrm{Z}+1 \mathrm{Que}$ se reduce $\mathrm{a}$ : $-\mathrm{X}-2 \mathrm{~W}+\mathrm{U}+3 \mathrm{Z}+1=0$

Es decir, esa es la relación que posibilita crear una estrella mágica con cinco variables. Para mostrar lo indicado, asignaremos a las variables, los siguientes valores: $X=-2, Y=-48, Z=-4, W=7 y$ $\mathrm{U}=-24$.

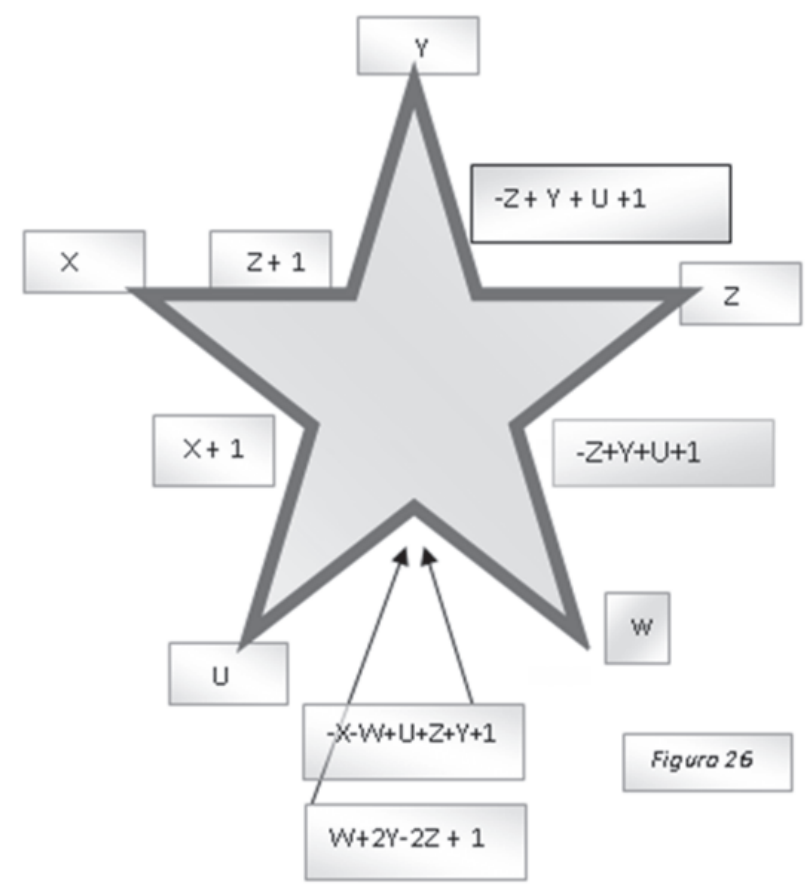

Fig. 24. Estrella mágica con cinco variables.

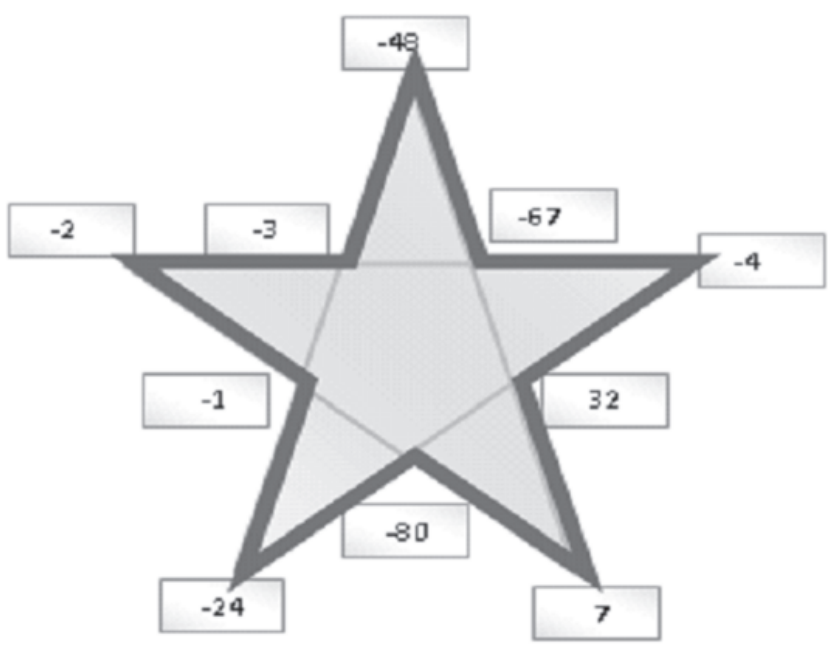

Fig. 25. Estrella mágica suma de segmento 76

Con estos valores, la constante de suma por segmento será -76 , y tendremos la estrella mágica representada en la Fig. 25, y si deseamos que todos los valores sean positivos, sumaremos a cada elemento la cantidad de 81, logrando una estrella mágica cuya constante es 248 , como se observa en la Fig. 26.

Rev. Ingeniería, Matemáticas y Ciencias de la Información Vol. 3 / Núm. 5 / enero-junio de 2016; pág. 79-97 


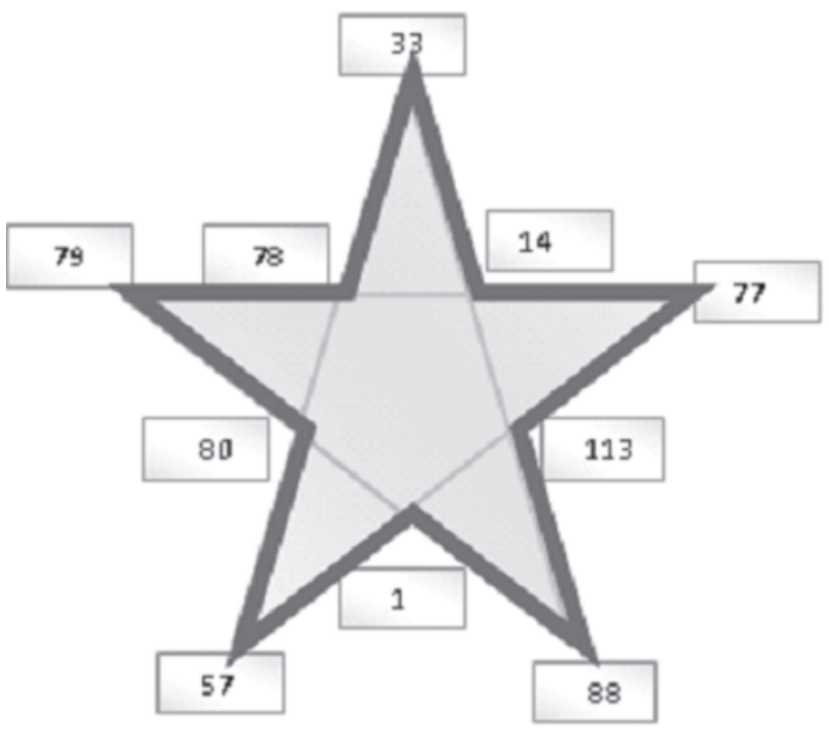

Fig. 26. Estrella mágica constante 248.

\section{E. Caso 7. Construcción de estrellas mágicas con seis variables}

El procedimiento es similar a los anteriores, y las limitaciones también, es decir deberemos buscar números diferentes que hagan que los elementos de cada segmento sumen una constante, en este caso ubicaremos además de en las puntas de las estrellas, donde ubicaremos: $X, Y, Z, W$ y U, en un corte ubicaremos la variable $\mathrm{T}$ como se observa en el ejemplo siguiente:

Ejemplo 9. En la Fig. 27, se establece los valores para cada uno de los elementos en función de las variables; X, Y, Z, W, U y T:

Construyendo de esa manera se establece que la constante de sumatoria por segmentos es: $\mathrm{T}+\mathrm{U}+\mathrm{W}+\mathrm{Y}+1$, obteniéndose además como condición para la construcción de este tipo de estrellas se obtiene: $-X+Z+W-U=-Y+Z+X+$ !,

Que se reduce a: $-2 X+Y+W-U-1=0$.

Obviamente que existen infinitas posibilidades de combinaciones de valores que cumplen la igualdad estipulada, proponemos la siguiente: $T=0, U=5$, $\mathrm{W}=6, \mathrm{X}=2, \mathrm{Y}=4, \mathrm{Z}=8$,

Que cumple la condición, debiendo indicar que $\mathrm{Z}$ y T, pueden tomar cualquier valor, ya que

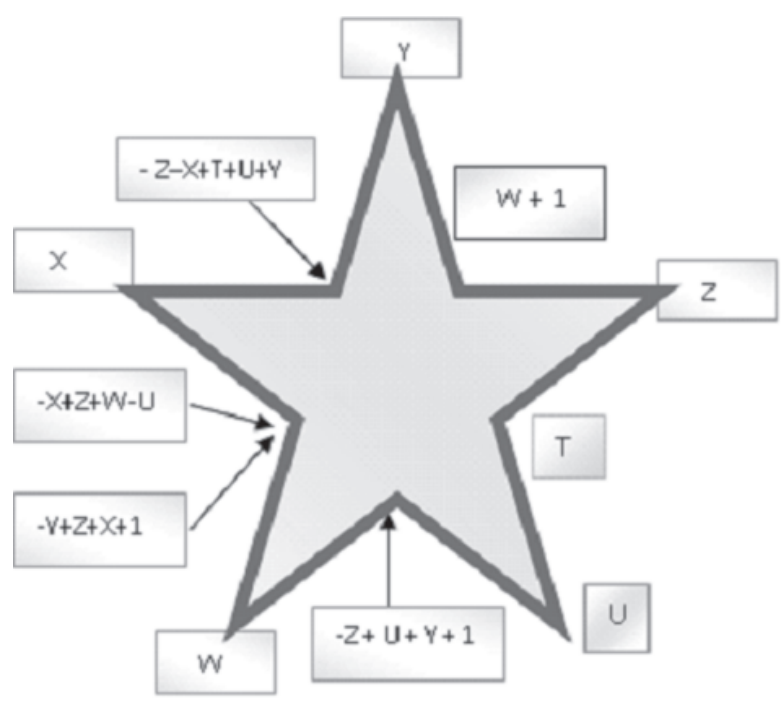

Fig. 27. Estrella mágica valores.

no forman parte de la condición expuesta. Con estos valores, tendremos la estrella mágica que se indica en la Fig. 28, que cumple las condiciones de estrella mágica, teniendo como sumatoria constante el valor de 34. En la misma existen valores negativos, si se desea que todos sean positivo, sumaremos a cada elemento el valor de 9 , dando por resultado, la estrella mágica de la Fig. 29, que tiene por constante de suma el valor de 70 .

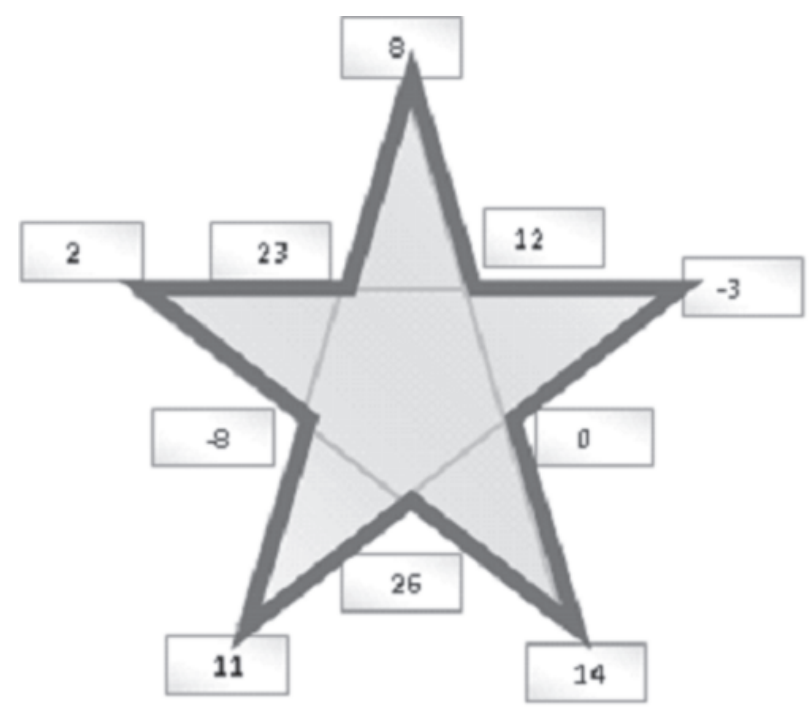

Fig. 28. Estrella mágica [34] y Fig. 29. Estrella mágica valores[70]. 


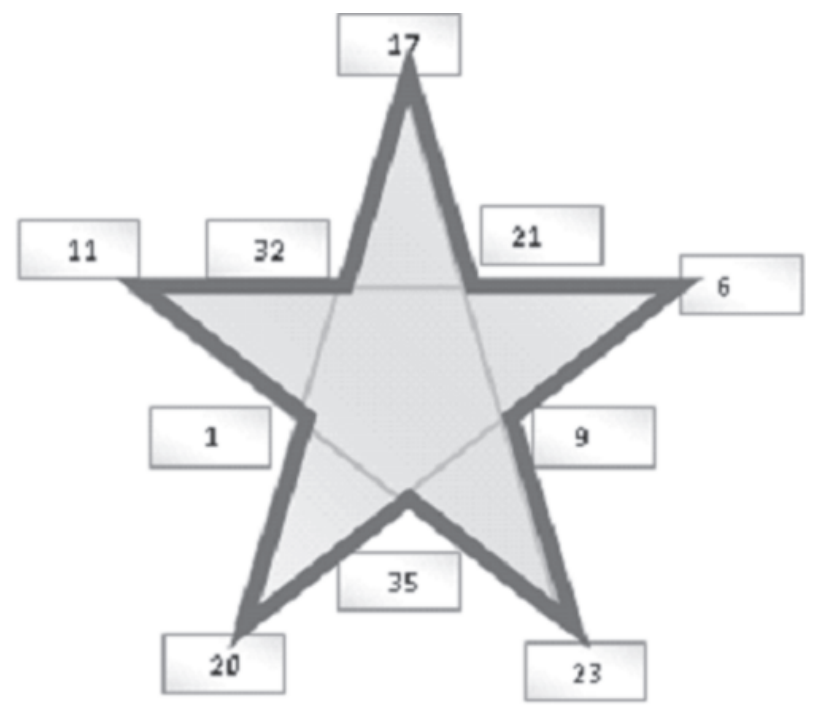

Fig. 29. Estrella mágica valores [70].

\section{F. Caso 8. Construcción de estrellas mágicas con siete variables}

Generalizando el procedimiento, fijaremos siete variables: T, U, V, W, X, Y, y Z, quedando libre únicamente tres elementos, en los cuales deberemos proceder a colocar valores, en función de las variables anotadas.

Ejemplo 10. Se establece directamente variables en las cinco puntas y en los dos puntos de corte, como se observa en la Fig. 30, inmediatamente se tiene que la constante de sumatoria es $\mathrm{U}+\mathrm{Z}+\mathrm{Y}+$ $X$, Con lo cual obtenemos valores para los otros elementos, en el primer caso- $\mathrm{T}-\mathrm{V}+\mathrm{Y}+\mathrm{X}+\mathrm{U}$, para el segundo caso tendremos $-\mathrm{U}+\mathrm{Z}+\mathrm{T}+\mathrm{V}-\mathrm{W}$ y para el ultimo elemento tendremos dos resultados, según los dos segmentos de los cuales forma parte, por un lado será: $2 \mathrm{U}-\mathrm{T}-2 \mathrm{~V}+\mathrm{Y}+\mathrm{W}$ y por otro: $\mathrm{U}+\mathrm{Z}+\mathrm{X}-\mathrm{W}-\mathrm{T}$, lo que obliga a que debamos trabajar la igualdad: $2 \mathrm{U}-\mathrm{T}-2 \mathrm{~V}+\mathrm{Y}+\mathrm{W}=\mathrm{U}$ $+\mathrm{Z}+\mathrm{X}-\mathrm{W}-\mathrm{T}$, Que reduciendo se tiene: $\mathrm{U}-\mathrm{Z}-$ $X-2 V+2 W+Y=0$, que constituye, para este caso la relación que debe existir entre las variables, cabe indicar que la variable $\mathrm{T}$, no forma parte de esa condición, por lo que podremos establecer su valor libremente.

Una de las combinaciones que posibilita construir la estrella mágica se da cuando asignamos los siguientes valores: $T=0, U=2, V=-4, W=1, X=-3, Y=6$ y $Z=21$.

Que genera la estrella de la Fig. 30, donde se tiene que los elementos de cada uno de los segmentos suman 26, y se tiene valores negativos, si la intención es que todos los elementos sean positivos, podríamos sumar a cada elemento el valor de 5, y se tendría la estrella mágica de la Fig. 31, donde la constante de suma en cada segmento es 46 .

Esta claro que esta no es la única, lo que aquí se plantea es únicamente una forma de construirlas,

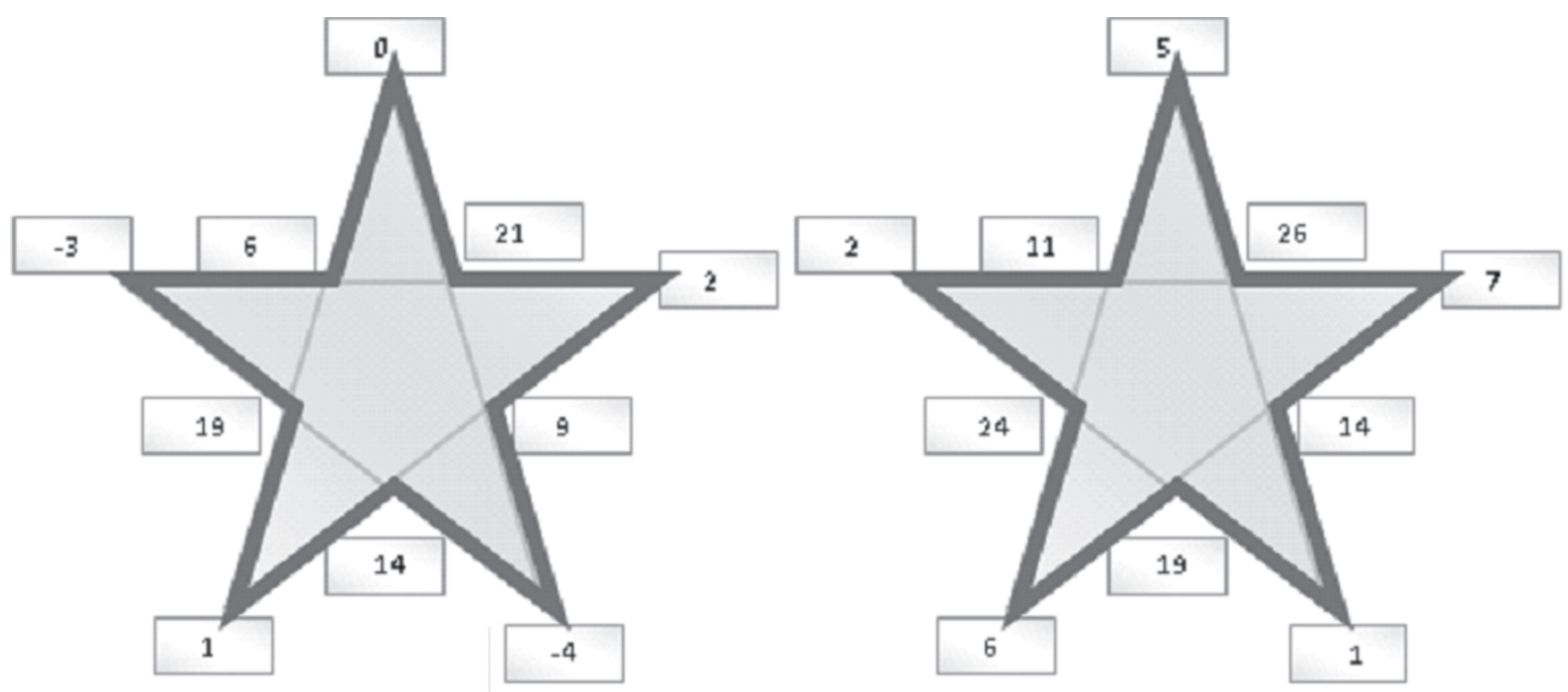

Fig. 30. Estrella valores [26] y Fig. 31. Estrella valores [46]. 
sabemos también que de cada una de ellas existen las generadas que son 12 , además por sumatoria $\mathrm{o}$ productos derivaran infinitas.

\section{G. Caso 9. Construcción de estrellas mágicas con ocho variables}

Generalizando el procedimiento, fijaremos ocho variables: S, T, U, V, W, X, Y, y Z, quedando libre únicamente tres elementos, en los cuales deberemos proceder a colocar valores, en función de las variables anotadas.

Ejemplo 11. Para este caso realizaremos dos ejemplos, ya que los mismos representan procesos distintos, el primero de ellos será colocando valores en las esquinas y los tres restantes en puntos de corte, dos de estos contiguos y el tercero saltado de los anteriores, las variables a ubicarse sobre los vértices serán: $S, T, U, V$ y $W$, en los cortes ubicaremos $\mathrm{X}, \mathrm{Y}$ e $\mathrm{Z}$, conforme se observa en la Fig. 32, estableciéndose como constante sumatoria por segmento el valor de $X+Y+W+T$, y quedando por ubicarse dos cortes, mas cada uno de ellos forma parte de dos segmentos por lo que en cada caso se establecerán condiciones ó sistemas de dos ecuaciones con dos incógnitas respectivamente. Asi en el un corte tenemos que con respecto al un segmento el valor será: $\mathrm{T}+\mathrm{W}+\mathrm{Y}-\mathrm{S}-\mathrm{U}$, y con respecto al otro segmento, el valor será: $-\mathrm{Z}-\mathrm{V}+\mathrm{X}+$ $\mathrm{Y}+\mathrm{W}$, de manera análoga, para el otro punto de corte, los valores serán $-Z-U+X+Y+T, y,-S-$

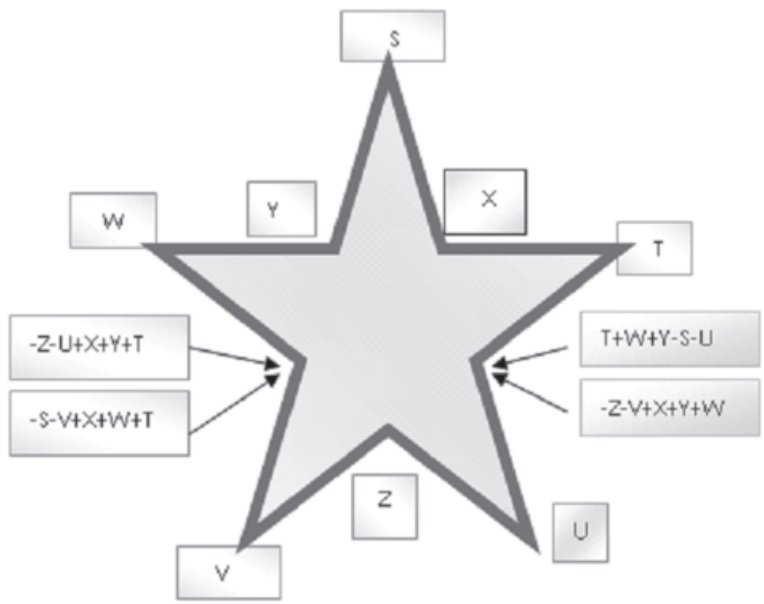

Fig. 32. Estrella mágica con ocho variables.

$\mathrm{V}+\mathrm{X}+\mathrm{W}+\mathrm{T}$, estableciéndose el siguiente sistema de ecuaciones:

$$
\left\{\begin{array}{l}
-Z-V+X+Y+W=T+W+Y-S-U \\
-Z-U+X+Y+T=-S-V+X+W+T
\end{array}\right.
$$

Que se reduce a:

$$
\left\{\begin{array}{c}
T+Z+V-X-S-U=0 \\
-Z-U+Y+S+V-W=0
\end{array}\right.
$$

Igualando las dos tendríamos que: $\mathrm{T}+\mathrm{Z}+\mathrm{V}-$ $X-S-U=-Z-U+Y+S+V-W$, que a su vez se reduce a:

$$
T+2 Z-Y-X+W-2 S=0
$$
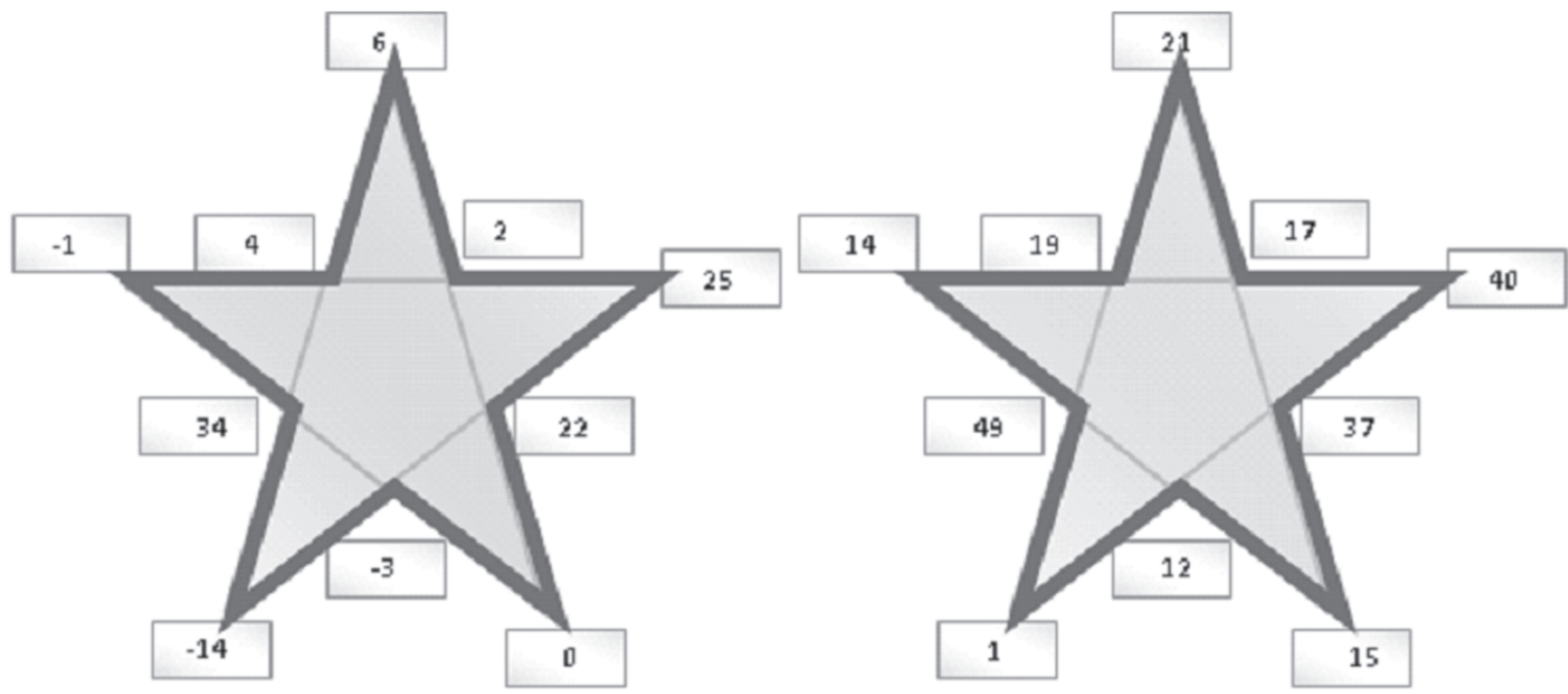

Fig. 33. Estrella mágica constante [30] y Fig. 34 Estrella mágica constante (90). 


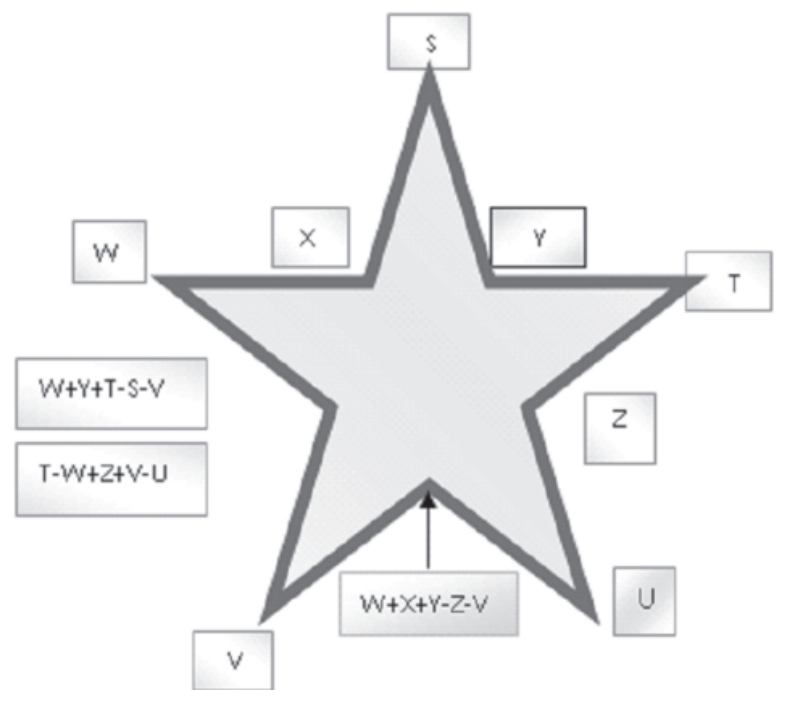

Fig. 35 Estrella mágica con nueve variables.

Se debe anotar que si despejamos V - U de cada ecuación del sistema se siente:

$$
\begin{aligned}
& V-U=S+X-T-Z \\
& V-U=Z+W-Y-S .
\end{aligned}
$$

Para solucionar, asignaremos entonces valores a cinco variables:

$X=2, Y=4, Z=-3, W=-1$ y $S=6$, lo que remplazando las ecuaciones anteriores da como resultados que: $\mathrm{T}=25 \mathrm{y} \mathrm{V}-\mathrm{U}=-14$.
La última relación brinda una infinidad de alternativas para $\mathrm{V}$ y $\mathrm{U}$, nosotros asignaremos los valores: $U=0$ y V $=-14$, con lo cual construimos la estrella mágica de la Fig. 36, cuya constante de suma por segmento es de 30.

De igual forma, si deseamos únicamente valores positivos, a cada elemento sumaremos la cantidad de 15, generando la estrella mágica de la Fig. 37 , que tiene constante de sumatoria por segmento igual a 90 .

\section{H. Caso 10. Construcción de estrellas mágicas con nueve variables}

Ejemplo 12. En este caso ubicaremos las variables en los vértices y en tres cortes consecutivos, como se observa en la Fig. 38, Debiendo indicar, que al igual que en el caso anterior, las variables que se asignen, deben someterse a restricciones de antemano.

Para este ejemplo colocaremos las variables $S$, $\mathrm{T} U, \mathrm{~V}$ y W en los vértices y $\mathrm{X}, \mathrm{Y}$ e $\mathrm{Z}$, en tres cortes consecutivos entre $\mathrm{U}$ y $\mathrm{W}$, como se observa en la Fig. 38, la condición de antemano en este caso sería que $\mathrm{W}+\mathrm{X}+\mathrm{T}=\mathrm{S}+\mathrm{Z}+\mathrm{U}$, la constante de suma sería entonces:

$$
W+X+Y+T
$$

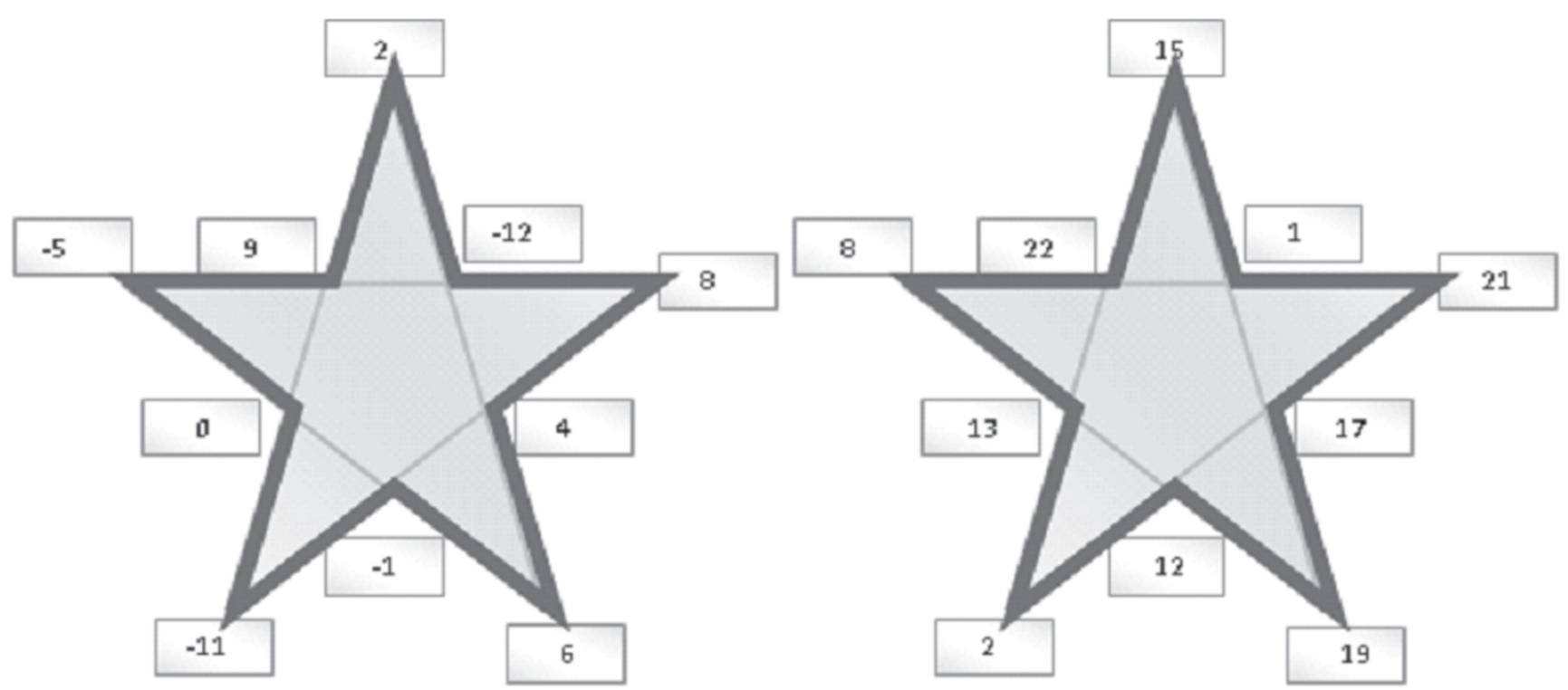

Fig. 36 Estrella mágica $Y=-12$ y $U=-11$ y Fig. 37 Estrella mágica valores enteros. 


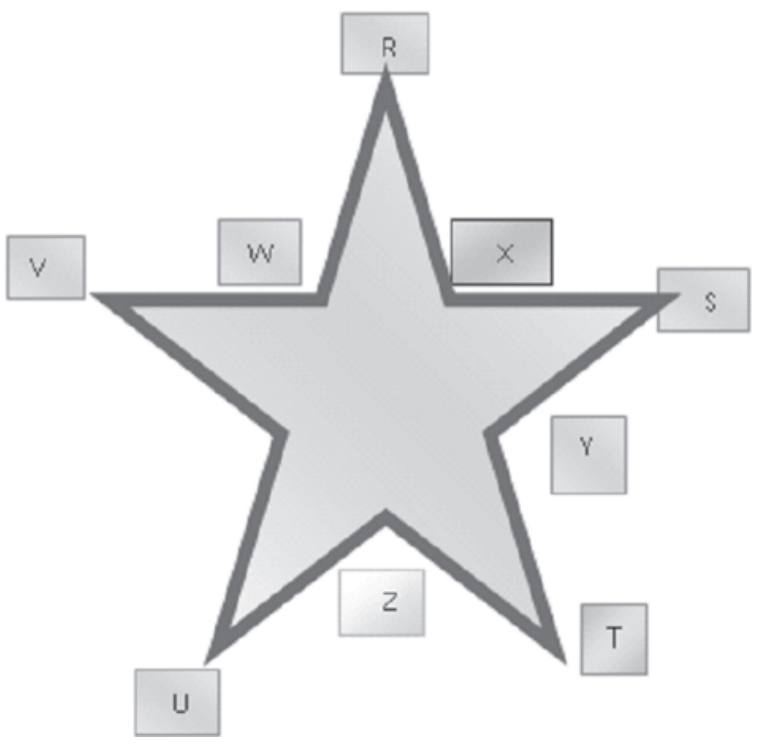

Fig. 38 Estrella mágica con variables en vértices.

Lo cual obligaría a que el siguiente punto de corte sea determinado por el valor de $\mathrm{W}+\mathrm{X}+\mathrm{Y}-$ $Z-V$. En el último punto de corte se establecerían dos valores, uno por cada segmento, $\mathrm{W}+\mathrm{Y}+\mathrm{T}-\mathrm{S}$ $-\mathrm{V}$, en el primer caso y $\mathrm{T}-\mathrm{W}+\mathrm{Z}+\mathrm{V}-\mathrm{U}$, lo cual genera la igualdad.

$\mathrm{T}-\mathrm{W}+\mathrm{Z}+\mathrm{V}-\mathrm{U}=\mathrm{W}+\mathrm{X}+\mathrm{Y}-\mathrm{S}-\mathrm{V}$, que reduciendo se obtiene la siguiente igualdad:

$$
-2 W+Z+2 V+S-U-Y=0
$$

Así, de las infinitas combinaciones, podríamos asignar valores para la igualdad que debe darse de antemano:

$$
S=2, Z=4, U=6, T=8, X=9, y W=-5,
$$

Que remplazando en la igualdad que se genera en el último punto de corte:

$10+4+2 \mathrm{~V}+2-6-\mathrm{Y}=0$, que reduciendo se obtiene: $2 \mathrm{~V}-\mathrm{Y}=-10$, relación lineal con infinitas duplas, asignaremos los siguientes valores: $\mathrm{Y}=-12$ y $\mathrm{U}=-11$.

Con los valores asignados tendremos la estrella mágica de la Fig. 36. Con una sumatoria constante, por segmento de 0 (cero).

Como en los demás casos si deseamos que los elementos sean todos positivos, sumamos 13 a cada uno y tendremos la Fig. 31, con una suma constante de 52 por segmento.
Ejemplo 13. Para este caso ubicaremos las variables en los vértices y en cuatro cortes, como se observa en la Fig. 38, Esta claro que tan solo un punto de corte estaría libre, además existen de inicio dos condiciones que deben cumplirse ya que tres segmentos están ya llenos, de antemano tenemos las igualdades de restricción:

$$
\left\{\begin{array}{l}
S+\mathrm{W}+\mathrm{V}=\mathrm{R}+\mathrm{Y}+\mathrm{T} \\
\mathrm{S}+\mathrm{Z}+\mathrm{U}=\mathrm{R}+\mathrm{X}+\mathrm{T}
\end{array}\right.
$$

Que puede expresarse de la siguiente manera:

$$
\left\{\begin{array}{l}
\mathrm{S}-\mathrm{R}-\mathrm{T}=\mathrm{Y}-\mathrm{W}-\mathrm{V} \\
\mathrm{S}-\mathrm{R}-\mathrm{T}=\mathrm{X}-\mathrm{Z}-\mathrm{U}
\end{array}\right.
$$

De donde se tiene que:

$Y-W-V=X-Z-U$, Que reduciendo es: $X=Y$ $+\mathrm{Z}+\mathrm{U}-\mathrm{W}-\mathrm{V}$

Que posibilita proponer infinitas combinaciones de valores, tomamos la siguiente:

$\mathrm{Y}=2, \mathrm{Z}=4, \mathrm{U}=-6, \mathrm{~V}=-3, \mathrm{~W}=-5$, que en base de la última igualdad da $X=8$.

Con lo cual retomando la igualdad de las relaciones iniciales se tendrá:

$$
\mathrm{S}-\mathrm{R}-\mathrm{T}=10 \text {, ó } \mathrm{R}+\mathrm{T}=\mathrm{S}-10 \text {. (a) }
$$

La constante sumatoria será: $V+W+X+S$.

Además obligadamente el elemento del corte sin valor debe cumplir a la ves dos igualdades, en función de la suma constante: $\mathrm{R}+\mathrm{W}+\mathrm{U}=\mathrm{V}+\mathrm{Z}+$ T. De donde se tiene: $R-T=-3+4+5+6=12$.

De donde se tiene que: $\mathrm{R}-\mathrm{T}=\mathrm{V}+\mathrm{Z}-\mathrm{W}-\mathrm{V}=$ $-3+4+5+6=12(b)$.

Con (a) y (b) se tendrá el siguiente sistema de ecuaciones:

$$
\left\{\begin{array}{c}
R+T=S-10 \\
R-T=12
\end{array}\right.
$$


Para resolver este sistema es necesario que asignemos un valor a $S$, proponemos $S=24$, que asegura que todos los elementos sean diferentes entre sí. Fig. 39 y 40.

\section{Cuya solución es $\mathrm{R}=13$ y $\mathrm{T}=1$}

Con lo cual hemos determinado todos los elementos de la estrella mágica que se observa en la Fig. 39. Esta estrella mágica tiene una constante de suma en sus segmentos que es: 24 . Si se desea que todos los elementos sean positivos, deberemos sumar 7 a cada elemento y obtendremos la estrella mágica que se observa el la Fig. 40, cuya sumatoria constante será igual a 52 .

Como en los demás casos esta es una solución de muchas que pueden obtenerse siguiendo este proceso, además de sus derivadas directas.

Este método, es sin duda el más general.

\section{Caso 11. Construcción de estrellas mágicas con diez variables}

Este caso es el que presenta mayor generalidad, ya que se asignará una variable a cada uno de los elementos, sin que ninguno de ellos quede libre, por tanto las relaciones aquí se establecen de antemano, en función de un segmento escogido en el cual se establecerá la constante de suma.
Ejemplo 14. Se establecen las diez variables, $Q$, $R, S, T, U, V, W, X, Y$ e Z. que se ubican en cada uno de los elementos de una estrella de cinco puntas, como se observa en la Fig. 41.

La constante para la suma será $Q+Y+W+S$

Como esta debe ser constante tendremos las siguientes igualdades.

a) $Q+W+S=U+Z+R$

b) $R+X+T=Q+V+S$

c) $S+Y+U=R+W+T$

d) $T+Z+Q=U+X+S$

e) $V . U+V+R=T+Y+Q$

Que constituyen un sistema de ecuaciones dependientes:

Entre la ecuación I y la II se tiene que: U+Z$\mathrm{W}=\mathrm{X}+\mathrm{T}-\mathrm{V}$

Entre la III y la IV se tiene que: $\mathrm{Z}+\mathrm{Q}-\mathrm{X}=\mathrm{R}+\mathrm{W}-\mathrm{Y}$

Relacionando estas dos se tiene que: $\mathrm{T}-\mathrm{V}-\mathrm{U}=\mathrm{R}-$ $\mathrm{Q}-\mathrm{Y}$, que es igual a: $\mathrm{T}+\mathrm{Q}+\mathrm{Y}=\mathrm{R}+\mathrm{U}+\mathrm{V}$, que es justamente la quinta ecuación, por ello y para buscar

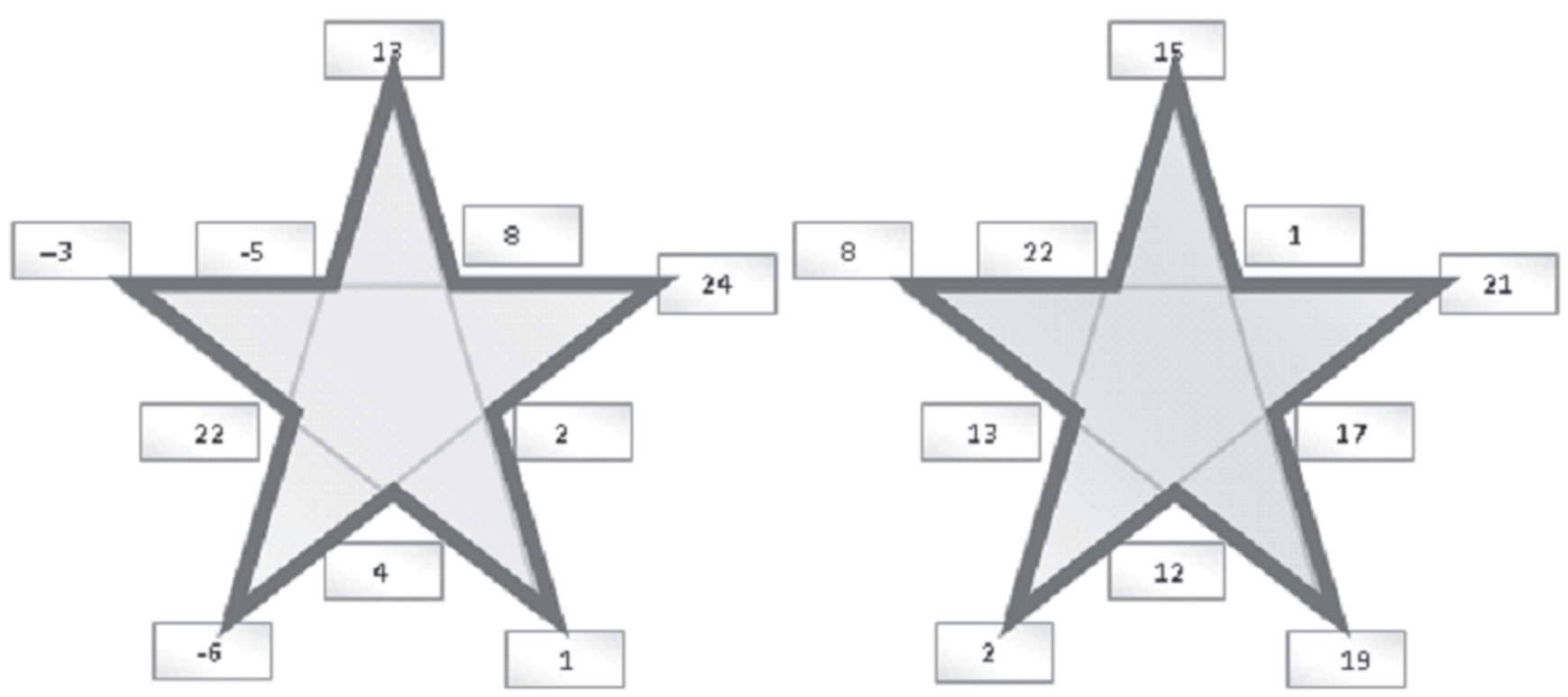

Fig. 39 Estrella mágica constante 24. Fig. 40 Estrella mágica constante 52. 


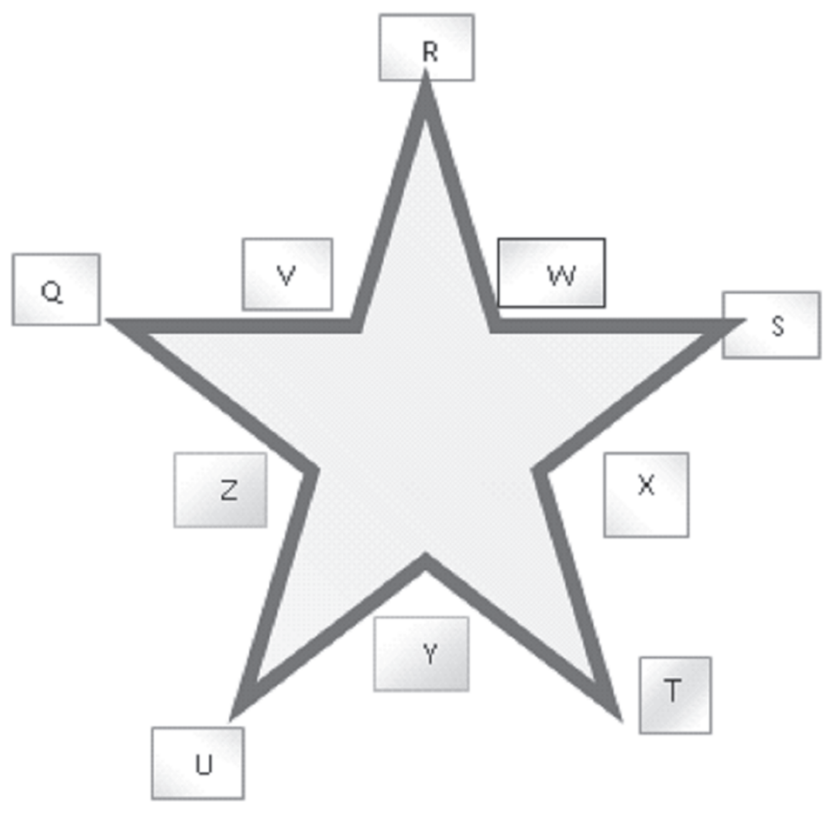

Fig. 41 Estrella mágica con diez variables.

solución trabajaremos únicamente con las primeras cuatro ecuaciones, con las que estableceremos el siguiente sistema de cuatro ecuaciones con diez incógnitas:

$$
\left\{\begin{array}{l}
Q+W+S=U+Z+R \\
R+X+T=Q+V+S \\
S+Y+U=R+W+T \\
T+Z+Q=U+X+S
\end{array}\right.
$$

Matemáticamente se sabe que es posible dar valores a seis variables para que el sistema tenga consistencia y brinde una solución única.

Para ello tomaremos la ecuación VI que resulta de relacionar la primera y la segunda:

VI. $U+Z-W=X+T-V$, que permite despejar una variable, y se tiene:

$$
\mathrm{U}=\mathrm{X}+\mathrm{T}-\mathrm{V}-\mathrm{Z}+\mathrm{W}
$$

Aquí podremos asignar ya cinco valores distintos, cuidando que el resultado además sea diferente a cada uno de los indicados, asignaremos:

$X=2, T=4, V=6, Z=-1$ y $W=-7$, lo que da como resultado $U=-6$.
Teniendo además que: $\mathrm{Q}+\mathrm{S}-\mathrm{R}=0$. (VII)

Igualando las ecuaciones III y IV, se tiene que: $\mathrm{R}+\mathrm{W}-\mathrm{Y}=\mathrm{Z}+\mathrm{Q}-\mathrm{X}$, que puede expresarse como:

$\mathrm{R}-\mathrm{Y}-\mathrm{Q}=\mathrm{Z}-\mathrm{X}-\mathrm{W}$, las variables del miembro izquierdo, tienen ya valores, asignándolos tendríamos:

$$
\mathrm{R}-\mathrm{Y}-\mathrm{Q}=-1-2+7=4(\mathrm{VIII})
$$

Relacionando VII y VIII, se tendría que: S - Y $=4$.

Aquí asignamos libremente el sexto valor $\mathrm{Y}=$ 10, y como consecuencia de la ecuación anterior se tendrá que $S=14$.

De la ecuación III, se tiene que: $\mathrm{R}=\mathrm{S}+\mathrm{Y}+\mathrm{U}-$ $\mathrm{W}-\mathrm{T}=14+10-6+7-4=21$

Y de la igualdad VI. Se tiene que $Q+14-21=0$. Entonces Q $=7$.

Con lo cual tenemos ya todos los valores, y la estrella mágica es la que se observa en la Fig. 42, que es una estrella mágica con suma constante por segmento de 20. Si aquí también deseamos que todos los elementos sean positivos deberemos sumar a cada elemento el valor de 8 y tendremos la estrella mágica de la Fig. 43, como sumatoria constante de 52.

\section{J. Caso 12. construcción de estrellas mágicas con diez variables donde la suma de los vertices es igual a la suma de los cortes}

Este caso es muy similar al anterior, debiendo añadirse la restricción de que los elementos de los vértices es igual a la suma de los cortes.

Ejemplo 15. Se establecen las diez variables, $Q$, $R, S, T, U, V, W, X, Y$ e $Z$. que se ubican en cada uno de los elementos de una estrella de cinco puntas, como se observa en la Fig. 41. Generándose el sistema siguiente:

$$
\left\{\begin{array}{c}
Q+R+S+T+U=V+W+X+Y+Z(V) \\
Q+W+S=U+Z+R \quad(I V) \\
R+X+T=Q+V+S \quad(I I I) \\
S+Y+U=R+W+T \quad(I I) \\
T+Z+Q=U+X+S \quad(I)
\end{array}\right.
$$



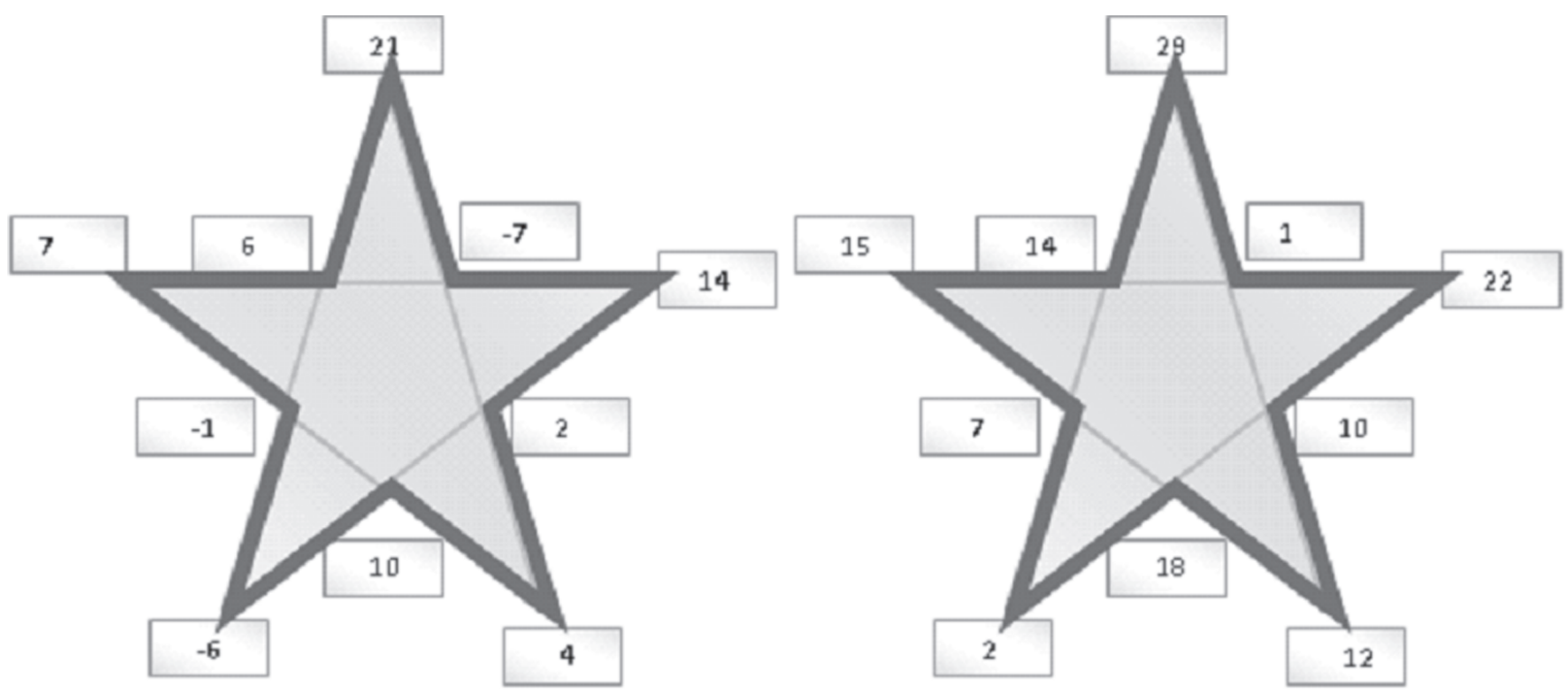

Fig. 42 Estrella mágica con suma constante por segmento de 20 y Fig. 43 Estrella mágica con suma constante por segmento de 52.

Donde la primera igualdad indica la restricción impuesta, las demás son iguales a las del caso anterior,

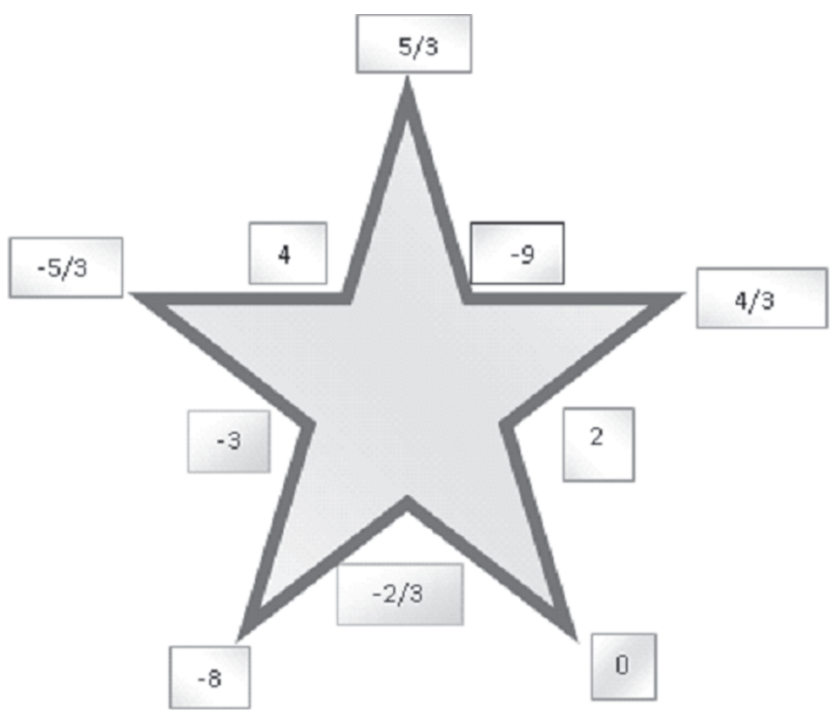

Fig. 44 Estrella mágica donde los elementos de cada segmento suman - 16 y los vértices y cortes suman -20.

Si igualamos IV y III se tendrá que: $\mathrm{U}+\mathrm{Z}-\mathrm{W}=$ $\mathrm{X}+\mathrm{T}-\mathrm{V}$, de donde $\mathrm{U}=\mathrm{X}+\mathrm{T}-\mathrm{V}-\mathrm{Z}+\mathrm{W}$.Asignamos los valores: $X=2, T=0, V=4, Z=-3$ y $W=-9$, que en base a la relación anterior resulta $U=-8$, se tiene también que: $\mathrm{Q}+\mathrm{S}-\mathrm{R}=-2$. (VII).
Además igualando las ecuaciones I y II, $\mathrm{R}+\mathrm{W}$ $\mathrm{Y}=\mathrm{Z}+\mathrm{Q}-\mathrm{X}$, entonces $\mathrm{R}-\mathrm{Y}-\mathrm{Q}=\mathrm{Z}-\mathrm{X}-\mathrm{W}$, que remplazando valores se tendrá: $\mathrm{R}-\mathrm{Y}-\mathrm{Q}=-3-2+$ $9=4$ (VII).

Relacionando VI con VII se tiene que: $\mathrm{S}-\mathrm{Y}=2$

Remplazando en la ecuación II, se tiene que: $\mathrm{R}$ $=\mathrm{S}+\mathrm{Y}+\mathrm{U}-\mathrm{W}-\mathrm{T}=\mathrm{Y}+2+\mathrm{Y}-8+9-0=2 \mathrm{Y}+3$

De VII, se tiene que $Q=R-S-2$, entonces: $Q=$ $2 Y+3-(Y+2)-2=Y-1$

Luego remplazamos en la primera igualdad: $Y$ $-1+2 Y+3+Y+2+0-8=4-9+2+Y-3$, que equivale a: $4 Y-4=Y-6$

Que reduciendo es: $3 Y=-2$, entonces, $Y=-2 / 3$

Consecuentemente: $\mathrm{S}=2-2 / 3=4 / 3, \mathrm{R}=2(-2 /$ 3) $+3=5 / 3, Q=-2 / 3-1=-5 / 3$.

Con base del procedimiento indicado se tiene una estrella mágica de cinco puntas, donde todos los elementos de cada segmento suman $-16 / 5$, y donde los vértices suman - 20/3 al igual que los cortes. Si a cada elemento se le multiplica por tres tendremos la estrella mágica de la Fig. 44, donde los elementos de cada segmento suman - 16 y los vértices y cortes suman -20.

Rev. Ingeniería, Matemáticas y Ciencias de la Información Vol. 3 / Núm. 5 / enero-junio de 2016; pág. 79-97 


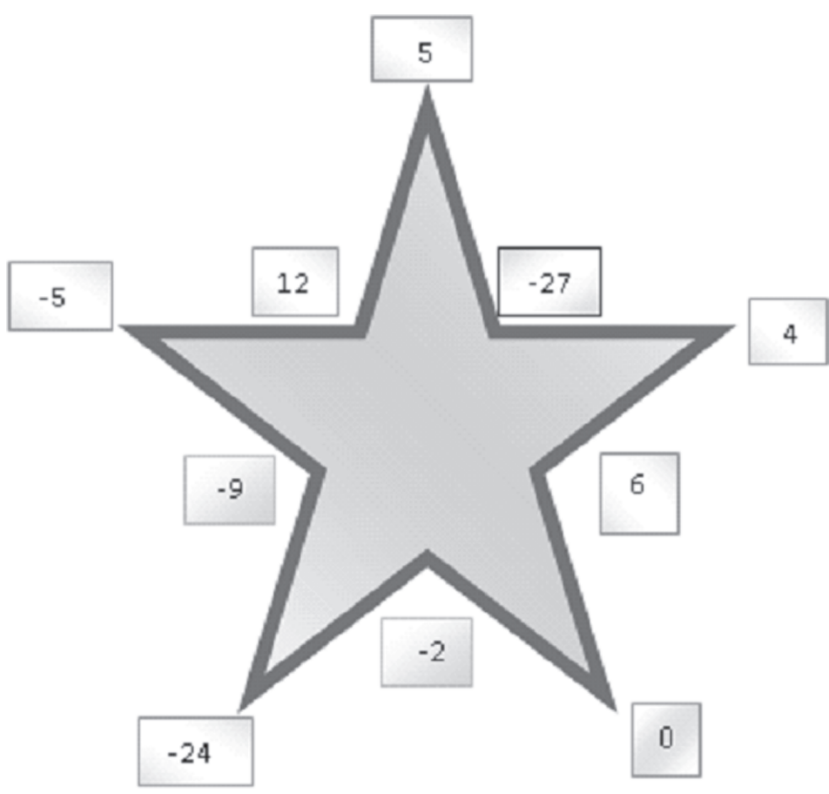

Fig. 45 Estrella mágica cuyos elementos, por segmento sumen 96 en, y cuyas aristas, al igual que cortes sumen 120.

Por último si se desea que todos los elementos sean positivos, deberemos sumar a cada uno de ellos, el valor de 28, con lo cual tendremos una estrella mágica, cuyos elementos, por segmento sumen 96 en, y cuyas aristas, al igual que cortes sumen 120.
NOTA: Esta restricción de que la suma de los vértices sea igual a la suma de los cortes, puede tratarse también en los demás casos, con procedimientos similares al expuesto, esto es, ingresando esa condición como una restricción más.

Los casos expuestos, no son en ningún caso los únicos, más bien, buscan proponer métodos y procesos de obtener estrellas mágicas de cinco puntas [6].

\section{REFERENCIAS}

[1] S. Fernández, Estrella mágica de cinco puntas. Ministerio de Educación y Ciencia (España), 2001.

[2] J.L.Adams, Guía y juegos para superar bloqueos mentales. Gredisa, Barcelona, 1999.

[3] M. De Alonso, Los juegos en el aula, Servicio de Publicaciones de CSI-CSIF. 2002.

[4] M. Gardner, Matemática para divertirse. Granica, Barcelona, 1988.

[5] I. Stewart, Ingeniosos encuentros entre juegos y matemática. Gredisa, Barcelona, 2000.

[6] R. Ramírez, El ingenio no tiene edad. Encuentro de profesores de matemáticas de Primaria y Secundaria, Castellón, 2003. 
